\title{
Dynamic mechanisms for pre-miRNA binding and export by Exportin-5
}

\author{
XIA WANG, ${ }^{1,2,5}$ XUE XU, ${ }^{1,2,5}$ ZHI MA, ${ }^{1,2}$ YINGQIU HUO, ${ }^{3}$ ZHENGTAO XIAO, $^{1,2}$ YAN LI, $^{4}$ \\ and YONGHUA WANG ${ }^{1,2,6}$ \\ ${ }^{1}$ Center of Bioinformatics, Northwest A\&F University, Yangling 712100, Shaanxi, China \\ ${ }^{2}$ College of Life Sciences, Northwest A\&F University, Yangling 712100, Shaanxi, China \\ ${ }^{3}$ College of Information Engineering, Northwest A\&F University, Yangling 712100, Shaanxi, China \\ ${ }^{4}$ Department of Materials Science and Chemical Engineering, Dalian University of Technology, Dalian 116023, Liaoning, China
}

\begin{abstract}
The biogenesis and function of mature microRNAs (miRNAs) is dependent on the nuclear export of miRNA precursors (premiRNA) by Exportin-5 (Exp5). To characterize the molecular mechanisms of how pre-miRNA is recognized and transported by Exp5, we have performed 21 molecular dynamic (MD) simulations of RNA-bound Exp5 (Exp5-RanGTP-premiRNA, Exp5RanGDP-premiRNA, Exp5-premiRNA), RNA-unbound Exp5 (Exp5-RanGTP, Exp5-RanGDP, apo-Exp5), and pre-miRNA. Our simulations with standard MD, steered molecular dynamics (SMD), and energy analysis have shown that (1) Free Exp5 undergoes extensive opening motion, and in this way facilitates the RanGTP binding. (2) RanGTP efficiently regulates the association/dissociation of pre-miRNA to its complex by inducing conformational changes in the HEAT-repeat helix stacking of Exp5. (3) The GTP hydrolysis prevents Ran from rebinding to Exp5 by regulating the hydrophobic interfaces and salt bridges between Ran and Exp5. (4) The transition from the $A^{\prime}$-form to the A-form of the pre-miRNA modulates the structural complementarities between the protein and the pre-miRNA, thus promoting efficient assembly of the complex. (5) The baseflipping process (from the closed to the fully flipped state) of the 2-nt $3^{\prime}$ overhang is a prerequisite for the pre-miRNA recognition by Exp5, which occurs in a sequence-independent manner as evidenced by the fact that different 2-nt $3^{\prime}$ overhangs bind to Exp5 in essentially the same way. And finally, a plausible mechanism of the pre-miRNA export cycle has been proposed explaining how the protein-protein and protein-RNA interactions are coordinated in physiological conditions.
\end{abstract}

Keywords: Exportin-5; miRNA; RanGTP; molecular dynamics

\section{INTRODUCTION}

MicroRNAs (miRNAs) are endogenous single-stranded RNAs (ssRNAs) of $\sim 21$ nt in length that play a key role as post-transcriptional regulators in eukaryotes by targeting mRNAs for cleavage and degradation (Bartel 2004). The biogenesis of miRNAs involves both nuclear and cytoplasmic processing events (Lee et al. 2002). The initial step is the precise nuclear cleavage of the long primary transcript (primiRNA) by a nuclear RNase III enzyme termed Drosha (Lee et al. 2003). This results in the formation of a 60- to 70-nt stem-loop intermediate that is known as the miRNA precursor (pre-miRNA) (Lee et al. 2002), bearing a 5' phosphate

\footnotetext{
${ }^{5}$ These authors contributed equally to this work.

${ }^{6}$ Corresponding author.

E-mail yh_wang@nwsuaf.edu.cn.

Article published online ahead of print. Article and publication date are at http://www.rnajournal.org/cgi/doi/10.1261/rna.2732611.
}

and a 2-nt 3' overhang (Lee et al. 2003). Additionally, premiRNAs are exported into the cytoplasm and are processed subsequently into $\sim 21$-bp miRNA duplexes by the cytoplasmic RNase III Dicer (Bernstein et al. 2001). Generally, only one strand of the duplex serves as the mature miRNA. This then loads to an Ago protein, the core constituent of the silencing effector complex RISC (RNA-induced silencing complex), in an asymmetric fashion (Schwarz et al. 2003), and thereby guides RISC to complementary mRNAs. More recently, we have investigated the molecular basis of miRNA interacting with the Ago protein and its target and have uncovered the key elements required for recognition of the miRNA target (Wang et al. 2010a). Furthermore, we have examined and quantified the dynamic properties, key rate-limiting steps as well as the stochastic noise that underlies the generic miRNA pathway (Wang et al. $2010 \mathrm{~b})$. In brief, these RNAs are generated in the cell nucleus but perform their functions in the cytoplasm and so need to be exported through nuclear pores into the 
cytoplasm. For pre-miRNA translocation in eukaryotes, Exp5 has been demonstrated as a Ran guanosine triphosphate (RanGTP)-dependent dsRNA-binding receptor that mediates the nuclear export of these pre-miRNAs (Yi et al. 2003; Lund et al. 2004; Zeng and Cullen 2004).

Nuclear transport occurs in several pathways through nuclear pore complexes (NPCs) that are large proteinaceous channels embedded in the nuclear membrane. Each pathway transports a specific range of macromolecules either into or out of the nucleus that are mediated by importins (import carriers) or exportins (export carriers) (Weis 2003). Pre-miRNA is exported by an evolutionarily conserved nucleocytoplasmic transport factor, Exp5. This not only serves as the nuclear export factor for pre-miRNAs but also protects pre-miRNAs from digestion by nucleases (Yi et al. 2003; Lund et al. 2004; Zeng and Cullen 2004). The pre-miRNA binding to Exp5 happens in a RanGTPdependent manner. This pre-miRNA/Exp5/RanGTP complex then migrates to the cytoplasm, where the release of pre-miRNA occurs in response to the hydrolysis of RanGTP to RanGDP, stimulated by RanGAP and other required cofactors (Kehlenbach et al. 1999). The ability of Exp5 to wrap around and release pre-miRNAs of different sizes and shapes with the help of Ran is essential for its transport function and requires a large degree of conformational flexibility.

Recently, the X-ray structure of the export complex of Exp5 bound to its cargo pre-miRNA-30a and RanGTP has been determined (PDB code: 3A6P) (Okada et al. 2009). In this ternary complex (Fig. 1A), Exp5 is a tightly wound U-like molecule constructed from an array of about 20 tandem HEAT repeats (Fig. 1C,D). Each HEAT repeat consists of a hairpin of two antiparallel $\alpha$-helices, designated A and B. The HEAT repeats are stacked to form an overall superhelical structure. The A helices form the outer convex surface (named "outer surface" for short), while the B helices form the inner concave surface (named "inner surface" for short). Inter-repeat linker loops and intra-repeat turns link the helices. Some of the linkers extend up to about 45 residues. At the bottom of Exp5, HEAT repeats 12 to 15 (H12-H15) form a tunnel-like substructure whose inner surface is positively charged. The Exp5-RanGTP complex forms a U-like structure to hold the pre-miRNA stem and the 2-nt $3^{\prime}$ overhang, thus protecting them from degradation. Further observation shows that the broadly distributed basic residues in the inner surface of Exp5 interact with the outer phosphodiester group of the pre-miRNA stem, while the tunnel of Exp5 and its adjacent loops contact with the 2-nt $3^{\prime}$ overhang through hydrogen bonds (H-bonds) and ionic interactions (Fig. 1D). Therefore, the recognition of premiRNA by Exp5 seems to be through this signature motif, i.e., the 2-nt 3' overhang (Yi et al. 2003; Lund et al. 2004; Zeng and Cullen 2004). This ternary complex provides a preliminary impression of nucleocytoplasmic transport of pre-miRNA. However, gaps still exist in our qualitative and

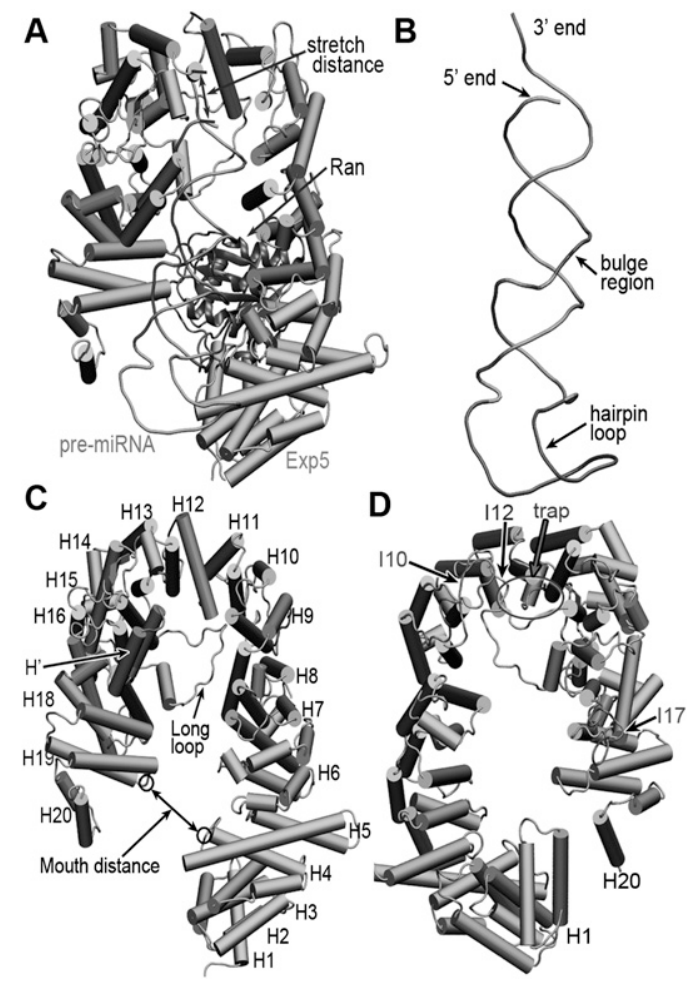

FIGURE 1. Overview structure of Exp5-GTP-RNA ternary complex. (A) The Exp5 superhelix is shown in the cartoon representation, the pre-miRNA is shown as the tube, and RanGTP is shown as the new cartoon. The stretch distance between pre-miRNA and Exp5 is defined as the change in distance between the center of mass (COM) of base pair G1/C61 and COM of H13B. (B) The reconstructed bulge region and hairpin loop in pre-miRNA are shown. $(C, D)$ The front and back views of the Exp5, respectively. Twenty consecutive HEAT repeats are labeled $\mathrm{H} 1-\mathrm{H} 20$ from the $\mathrm{N}$ terminus (H1) to the $\mathrm{C}$ terminus (H20). Each HEAT repeat consists of a hairpin of $\alpha$-helices and a linker. The trap region comprised of I10, I12, I13, "Long loop," and H12-H15 is highlighted in ellipse. The "Mouth distance" is defined as the distance between COM of Gln168 and COM of His 1086.

quantitative understanding of the thermodynamic and kinetic mechanisms of this pathway:

1. This tightly wound U-like molecule is expected to be intrinsically flexible (Okada et al. 2009), but how does the conformational change of the protein occur, which is crucially important for both the cargo binding and release?

2. How does Exp5 recognize and protect the pre-miRNA dynamically?

3. Are there conformational changes of pre-miRNA that facilitate the ternary structure assembly and, if so, how?

4. How does RanGTP cooperate with the binding of premiRNA dynamically?

5. What conformational events and changes prevent the functionally adverse reassociation of Exp5 with RanGDP or pre-miRNA in the cytoplasm, ensuring a highperformance and one-way transition? 
Efforts to fill these gaps have been hampered by limited understanding of the knowledge of the structures and the dynamics of different conformations including the free Exp5 and pre-miRNA- and RanGTP-bound Exp5s. Moreover, functional dynamics in macromolecular systems are difficult to investigate experimentally and are impossible to determine simply by observing crystal or NMR structures. Therefore, theoretical techniques, such as molecular dynamic simulations that serve as a link between structure and dynamics by providing detailed atomic motions as a function of time, have unique advantages pointing at the problems we mentioned above (MacKerell and Nilsson 2008; Wang et al. 2010a).

In this study, we investigated these issues by performing all-atom multi-nanosecond molecular dynamics (MD) simulations of Exp5 in aqueous solution. Our simulations provide a time-resolved atomistic and causal picture of these pronounced structural transitions, facilitating the understanding of the thermodynamic and kinetic puzzles of nucleocytoplasmic transport of pre-miRNA. Besides normal MD simulations, steered molecular dynamics (SMD) are also used to confirm the cooperative mechanism between RanGTP and pre-miRNA.

\section{RESULTS}

\section{Intrinsic and ligand-induced flexibility}

To provide a measure of the relative flexibility of different regions of the Exp5 models, the root mean square fluctuations (RMSFs) of $\mathrm{C}_{\alpha}$ atoms with respect to their timeaveraged positions are presented in Figure 2. The RMSF profiles show that the five systems share a zig-zag pattern that is similar to importin- $\beta$ 's such as Transportin-1 (data not shown). The most rigid regions are located in the $\alpha$ - and $\beta$-folded regions $(\sim 0.8 \AA)$, whereas the flexible parts $(\sim 2.5 \AA)$ are mainly in loops that are oriented away from the rest of the protein, toward the surrounding solvent.

In apo-Exp5, since there was no ligand bound to the $\mathrm{N}$ and $\mathrm{C}$ termini of Exp5, both regions show significantly

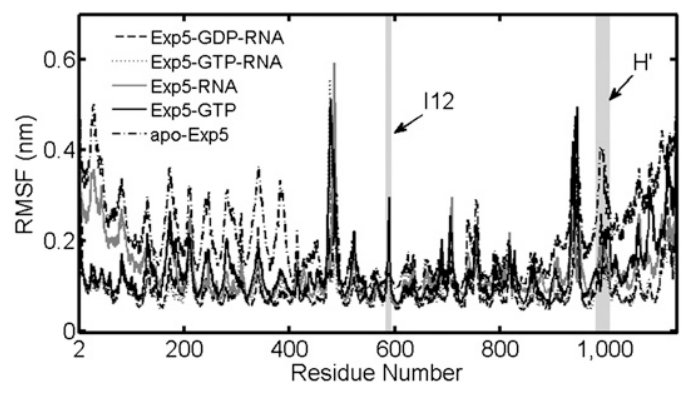

FIGURE 2. The RMSFs (in nanometers) of the $\mathrm{C}_{\alpha}$ atoms around their average positions during the simulation time. The graph is a superposition of five curves (corresponding to the five Exp5 related simulations) with the horizontal axis corresponding to the residue numbers. $\mathrm{I} 12$ and $\mathrm{H}^{\prime}$ highlighted in shadow. higher fluctuations than those of central regions $(\sim 5 \AA$ for the former vs. $\sim 1.9 \AA$ for the latter). Compared with apoExp5, the bound Exp5s have lower fluctuations, indicating that the presence of pre-miRNA and/or Ran can stabilize Exp5. For example, in Exp5-GTP simulation, the reduced mobility of the residues is clearly visible in the $\mathrm{N}$ and $\mathrm{C}$ termini of Exp5 relative to those of apo-Exp5 $(\sim 1.6 \AA$ for the bound system vs. $\sim 5 \AA$ for the apo system), particularly for the main binding sites of Ran, H1-H9 (residues 2-460 in Fig. 2; Okada et al. 2009). It is suggested that the saltbridging interactions (for details, see "GTP Hydrolysis Undermines the Reassembly of Ran to Exp5 in Cytoplasm" below), as well as the electrostatic complementarities in the interfaces between Ran and Exp5, significantly constrain the curvature of $\mathrm{H} 1-\mathrm{H} 9$, producing a stable core in the complex.

In Exp5-RNA simulation, the binding of pre-miRNA largely reduces the fluctuations of residues H4-H20 (RMSF $=\sim 1.1 \AA$ ), a region strongly correlated with the motions of the stem pre-miRNA due to the electrostatic interactions between them (data not shown). Despite the rigidity of Exp5 in Exp5-RNA, residues H1-H3 (residues 2-121 in Fig. 2) in this system still display relatively high RMSF values $(\sim 2.3 \AA)$, comparable to those in apo-Exp5, indicating that the $\mathrm{N}$ terminus of Exp5 is unstable. As for Exp5-GTP-RNA and Exp5-GDP-RNA, both $\mathrm{N}$ and C termini of Exp5, particularly for $\mathrm{H} 1-\mathrm{H} 3$ and $\mathrm{H} 18 \mathrm{~B}-\mathrm{H} 20$ (residues 1032-1136 in Fig. 2), show high stability $(\sim 1.1 \AA$ ) since they are complexed with binding partners. Comparison of all the four bound Exp5 simulations reveals that the global mobility of the $\mathrm{N}$ and $\mathrm{C}$ termini of Exp5 is structurally dependent, while the central region is independent of the presence or absence of Ran/pre-miRNA, with the exception of loop I12 (a loop connecting H12A and H12B, residues 582-595) (Fig. 1D).

I12, as an element of the trap that holds the 2-nt $3^{\prime}$ overhang (Fig. 1D), displays much lower RMSF values $(\sim 1.6 \AA$ on average $)$ in apo-Exp5 than in other systems, due to the hydrogen bonds (H-bonds) formed by Glu588 (in I12) with Lys830 (in the "Long loop") and Arg598 (in H12). In the Exp5-GTP system, however, with the H-bond disruption induced by the binding of RanGTP, I12 is released and becomes more flexible $(\sim 2.1 \AA)$. Thus, it has a greater chance to "grasp" the 3 ' end of pre-miRNA. Once the pre-miRNA bound to the Exp5, I12 is locked by a set of H-bonds contributed by the 2-nt $3^{\prime}$ overhang of pre-miRNA and becomes more stable, as shown by the smallest RMSF of 1.5-1.7 $\AA$ in Exp5-GTP-RNA, Exp5GDP-RNA, and Exp5-RNA systems.

In addition, Figure 2 shows some interesting features of insert $\mathrm{H}^{\prime}$ (Fig. 1C), a reconstructed HEAT repeat that connects H17B and H18A. In the apo-Exp5 system, this region has a higher RMSF value $(\sim 5.0 \AA)$ compared with those in the other complexes. The PCA analysis (for details, see the next section, "Free Exp5 Transits to the Extended 
State") further shows that $\mathrm{H}^{\prime}$ presents a simple pendulum motion away from the tunnel elements, which makes it fully exposed in solvent (Supplemental Fig. S1A,B), implying that the large fluctuations of unbound $\mathrm{H}^{\prime}$ should be of functional significance, i.e., increasing the possibility of $\mathrm{H}$-bond formation with pre-miRNA. Interestingly, once RanGTP binds Exp5, $\mathrm{H}^{\prime}$ immediately rotates $\sim 70^{\circ}$ and approaches the tunnel from its initial free state (Supplemental Fig. S1C,D). In this case, the $\mathrm{H}^{\prime}$ is stabilized (average $\mathrm{RMSF}=1.7 \AA$ ) by $\mathrm{H} 12-\mathrm{H} 15$ by the electrostatic attractions between them. For all the pre-miRNA binding complexes, $\mathrm{H}^{\prime}$ produces three $\mathrm{H}$-bonds, with one involved in Ser986 and C13 of pre-miRNA and two others belonging to Glu1002-Ser756 and Met 998-Asn758; these interactions result in stabilization of the complexes, which further enhances the communication between pre-miRNA and Exp5 (Supplemental Fig. S2).

\section{Free Exp5 transits to the extended state}

To characterize the overall shape of the Exp5 conformations in the apo-Exp5 simulation, the radius of gyration ( $\mathrm{Rg}$ ), defined as the mass-weighted RMSD of a collection of atoms from their common center of mass, is used and analyzed for three apo-Exp5's along their simulation trajectories (Fig. 3; Supplemental Fig. S3). The curves corresponding to these three 45-nsec apo-Exp5 simulations oscillate in a large range from 3.66 to $4.3 \mathrm{~nm}$, indicating that the free Exp5 in solvent is flexible and has a considerably extended conformation. Over the course of the simulation, the end-to-end distance is increased by $23 \%$ (from 5.6 to $6.9 \mathrm{~nm}$ ), as measured from the geometric centers of Phe36 and Gln1128 at two ends (Supplemental Fig. S4).

In comparison with apo-Exp5, one Ran-unbound (Exp5-RNA) and three Ran-bound systems (Exp5-GTP, Exp5-GTP-RNA, and Exp5-GDP-RNA) were also simulated and examined (Fig. 3). The Ran-bound systems show structural stability indicated by the smaller $\operatorname{Rg}(\sim 3.68 \mathrm{~nm})$,

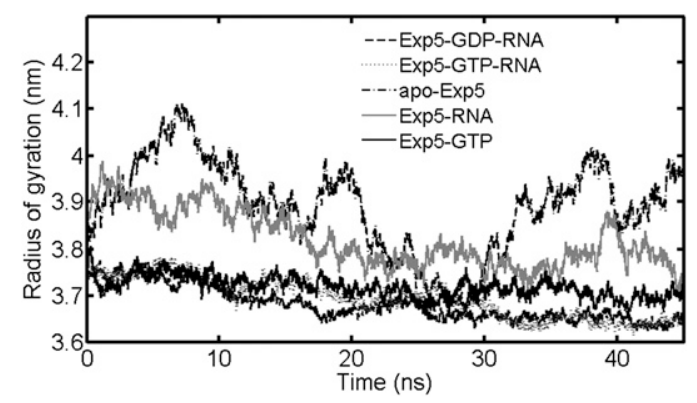

FIGURE 3. The time evolution of the radius of gyration ( $\mathrm{Rg}$ ) of $\mathrm{C}_{\alpha}$ (in nanometers) with respect to the starting structure during molecular dynamics simulations is shown for apo-Exp5, Exp5-GTP, Exp5-RNA, Exp5-GTP-RNA, and Exp5-GDP-RNA complexes. suggesting that the binding of pre-miRNA and Ran makes Exp5 rigid and more compact. This result is further supported by measuring the U-holder "mouth distance" (the COMs of Gln168 and His1086 are taken as reference) (Fig. 1B). The free Exp5 opens its "mouth" widely to 2.64 $\pm 0.48 \mathrm{~nm}$, and Exp5-GTP becomes slightly narrower $(2.04 \pm 0.18 \mathrm{~nm})$, while Exp5-GTP-RNA is closed to the greatest extent $(1.38 \pm 0.13 \mathrm{~nm})$ (Supplemental Fig. S5).

To better understand the global motions of free Exp5, the molecular dynamics trajectory was analyzed by only examining the motions along the Principal Components. Application of Principal Components Analysis (PCA) to the $\mathrm{C}_{\alpha}$ atom motions of the simulation indicates that $>82 \%$ of these motions are accounted for by the first three eigenvectors. Accordingly, the projection of the structures of the $\mathrm{C}_{\alpha}$ atoms in the MD trajectories onto the essential space (planes) defined by PC1/PC2, PC1/PC3, and PC2/ PC3 is illustrated in Figure 4, which allows one to visualize the conformational spaces sampled from MD calculations. The size of each cluster in Figure 4 appears to indicate that apo-Exp5 undergoes large conformational changes during simulation. The trajectory of the apo-Exp5 simulation projected onto PC1/PC2 and PC1/PC3 has two well-defined clusters, indicating that this system samples two distinct minima during the molecular dynamics trajectory (Fig. 4).

To characterize the collective motions represented by the first three dominant eigenvectors, DYNDOM analysis was used to identify the corresponding motion modes. The results showed that the transition of free Exp5 involves the rotation and bending motions of $\mathrm{H} 1-\mathrm{H} 8$ and $\mathrm{H} 19 \mathrm{~B}-\mathrm{H} 20$ (Fig. 5), consistent with the RMSF analysis that both $\mathrm{N}$ and $\mathrm{C}$ termini of Exp5 undergo high fluctuations in the absence of Ran and pre-miRNA (Fig. 2).

PC1 displays a pivotal rotation of $\mathrm{H} 1-\mathrm{H} 8$ relative to $\mathrm{H} 9-$ $\mathrm{H} 20$, which will distort and expose the basic inner surface of Exp5 to the solution. This large-scale outward motion prevents pre-miRNA from rebinding to Exp5 when the complex disassociates in the cytoplasm (Fig. 5A). PC2 of free Exp5 largely corresponds to the motions of $\mathrm{H} 1-\mathrm{H} 8$ and H19B-HR20, which rotate in an opposite direction to the least flexible segment H9-H19A. This is suggestive of a mechanism that the coupling of motions between N-terminal (H1-H8) and C-terminal (H19B-H20) domains opens the U-like mouth of Exp5 in Exp5-GTP-RNA. This is further supported by the analysis of fluctuation of the eigenvalues of this component, which is well consistent with the conformational opening profile of free Exp5 (Fig. 5B; Supplemental Fig. S6). This opening motion makes RanGTP (especially for switches I and II) more accessible to its inner surface, eventually resulting in an intimate contact between Exp5 and RanGTP. Thus, apo-Exp5 opens to the largest extent that is beneficial for the easy recruitment of RanGTP in the nucleus (Supplemental Fig. S4). PC2 also reveals that $\mathrm{H}^{\prime}$ moves toward the bottom of Exp5, which is 

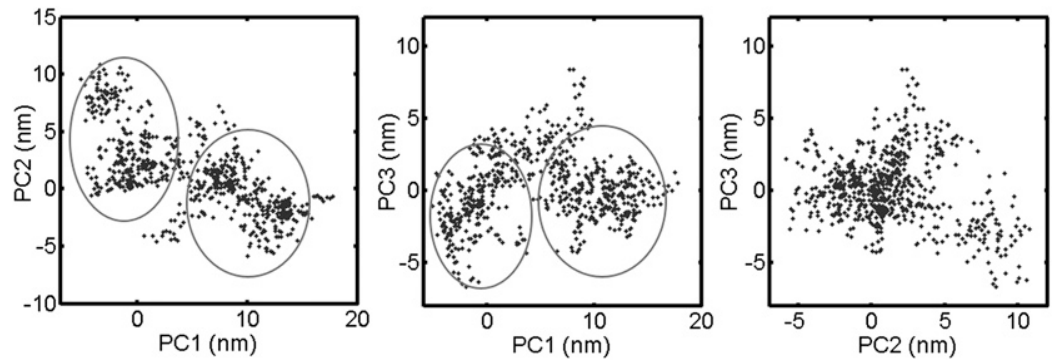

FIGURE 4. $2 \mathrm{D}$ projection of $\mathrm{MD}$ structures onto the plane defined by the top three eigenvectors from PCA. The cloud represents all three trajectories of apo-Exp5, projected onto the first three eigenvectors. Every sixth frame of the respective trajectories has been used in the projections.

probably relevant to the pre-miRNA binding. PC3 represents a significantly smaller displacement of atoms and shows quite similar results as PC1 and is not discussed here to save space (Fig. 5C). Therefore, on the basis of the nature of the dominant modes of conformational changes in the apo-Exp5 system, two different major regions- $\mathrm{H} 1-\mathrm{H} 8$ and $\mathrm{H} 9-\mathrm{H} 20$ - are clearly distinguished in Exp5 structure. The large-scale motions of these finally make apo-Exp5 transit into an extended U-like conformation (Supplemental Figs. S1, S4).

\section{Water distribution analysis of the inner and outer sides of free Exp5}

Water, which always appears at the surface of biological macromolecules and self-organized assemblies, plays a pivotal role in the structure, stability, and function of these systems (Nandi et al. 2000; Teeter et al. 2001). It has long been recognized to be indispensable in protein-nucleic acid association (Schwabe 1997) and enables them to be identified by structural and computational analyses (Reddy et al. 2001; Duan and Nilsson 2002; Lynch et al. 2002). As to Exp5, it is a soluble cellular export receptor that has exposed binding sites in an aqueous environment. Thus, in the present study, to give insights into the mechanism of assembly for cargoes-Exp5 during pre-miRNA export pathway, we analyzed the distribution and the dynamical behavior of water around Exp5 using the methods described in the Materials and Methods section.

As shown in Figure 6, we have identified the mobility of water on the global surface of Exp5 derived from the apoExp5 trajectory. Overall, the water shell follows the shape of the protein, and the spatial distribution of water molecules is both highly asymmetric and anisotropic. Closer inspection reveals three intriguing findings:

1. The high-density region is located on the inner surface of the protein, and the low-density areas at the outer surface (Fig. 6A). This phenomenon suggests a hydrophilic nature of the inner surface of Exp5 and charac- terizes hydrophobicity of the outer surface that facilitates the binding of Exp5 to the active sites on the surface of NPC.

2. The water density at the inner surface of the central part of Exp5 is significantly higher than that at the $\mathrm{N}$ and $\mathrm{C}$ termini (Fig. 6A). In other words, more water molecules are observed in the regions of major nucleotide binding surfaces (B helices of H8-H17 of Exp5) than in the Ran binding sites (B helices of $\mathrm{H1}-$ H7 and H18-H19) and H20. Such an asymmetric distribution of water would principally buffer the electrostatic repulsions between phosphate groups of RNA and the electronegative atoms on the protein (Reddy et al. 2001). For the Ran binding surfaces, the lower hydrophilic nature suggests that hydrophobic interactions play important roles in RanGTP binding to Exp5 (Phillips 2008).

3. The highest-density region of water resides at the tunnel of Exp5 (H12-H15), indicating that the deep concaves on the surface of the moving side chain can capture more solvent molecules than the two sides' inner surfaces (B helices of $\mathrm{H} 1-\mathrm{H} 11$ and $\mathrm{H} 16-\mathrm{H} 20$ ) (Fig. 6A). This result qualitatively agrees with the report by Phillips and Pettitt (1995). Compared with the tunnel of Exp5, the solvent density around the linkers of Exp5 appears much weaker (Fig. 6B), except for the trap region (Fig. 6B) that forms a hydrophilic pocket to accommodate the $3^{\prime}$ overhang of pre-miRNA. However, waters in the trap are kinetically labile and exchange quickly with the bulk, with maximum residence times of $<100$ psec. This behavior of labile hydration structures probably originates from local side-chain movements.

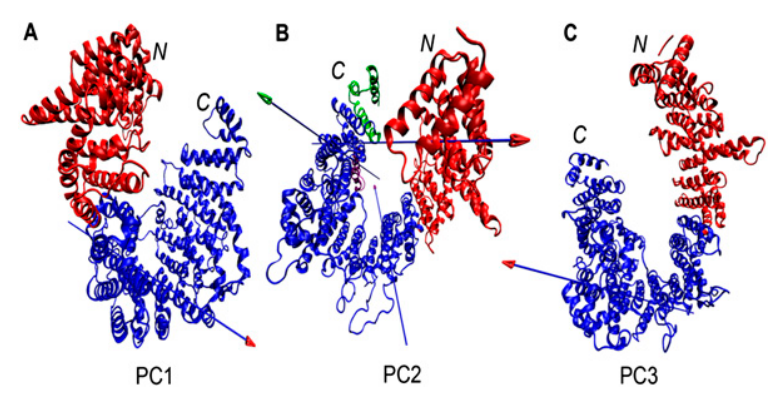

FIGURE 5. Principal components of free Exp5 dynamics. (Arrows) The axes of motion. The colors of the arrow shaft and head correspond to the colors of the static and dynamic domains, respectively. Both PC1 $(A)$ and PC3 $(C)$ describe a rotation motion of $\mathrm{H} 1-\mathrm{H} 8$ and H9-H20. PC2 (B) represents conformational opening motions and corresponds to the motions of $\mathrm{H} 1-\mathrm{H} 8$ and H19B-HR20, which rotate in opposite direction to the least flexible segment H9$\mathrm{H} 19 \mathrm{~A}$, and the motion that $\mathrm{H}^{\prime}$ moves toward the bottom of Exp5. 


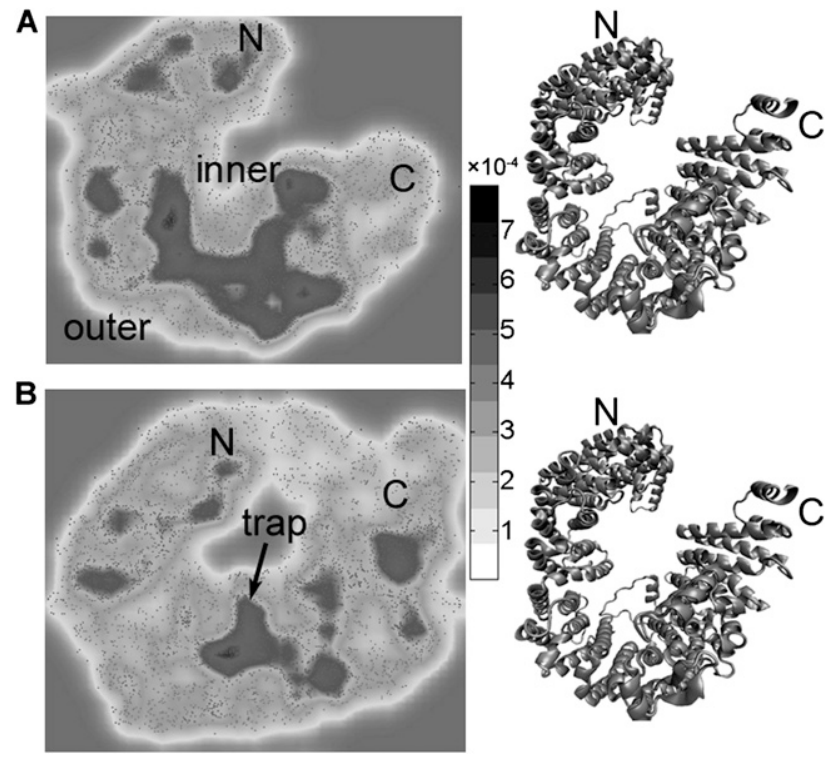

FIGURE 6. Contour maps of water density distribution around the free Exp5 surface. Water molecules are taken from the whole Exp5 surface $(A)$ and all linker loops of $\operatorname{Exp} 5(B)$. The radius was set at 1.8 $\AA$. (Left side) Fifty percent of the density in a quantitative plot. All the images are displayed in the logarithmic scale (base 10) for clarity of the presentation. (Right side) The protein cartoon structure, which is directly taken out and shown in the same coordinates as that in water (left side).

\section{Exp5 recognizes the pre-miRNA through the base flipping of the 2-nt $3^{\prime}$ overhang and widening of the minor groove of the stem}

To explore the mechanism of pre-miRNA recognition by Exp5, we examined the conformational changes of the free pre-miRNA in solution in comparison with the bound premiRNA in Exp5-GTP-RNA.

For the free pre-miRNA, the helical structure is linearly extended and maintains stability (RMSF $=\sim 2.5 \AA$ ) over the entire simulation, with the bulge and loop residues fluctuating strongly. Especially, the 2-nt $3^{\prime}$ end undergoes high fluctuations and is very unstable (RMSF $=\sim 7.8 \AA$ ) in the initial $10 \mathrm{nsec}$, making free pre-miRNA an "open" state and allowing the 2-nt $3^{\prime}$ overhang to be fully exposed in solution. Surprisingly, after $10 \mathrm{nsec}$, a flipping upward motion occurs for the $3^{\prime}$ end, which pushes the terminal bases close to the RNA minor groove and the sugarphosphate backbone exposed in solution (Fig. 7). Such a fully "closed" state is quite stable in the last $35 \mathrm{nsec}(75 \%$ of the simulation time), indicating that this conformation is a free energy minimum for the free pre-miRNA in solution and is of functional significance because the relatively low steric barrier posed by the closed state makes the $3^{\prime}$ end be easily trapped by Exp5. In addition, the projection of the dynamics of the free pre-miRNA onto its bound coordinates in Exp5-GTP-RNA shows that the binding process entails a transition of the 2 -nt 3 ' overhang from the closed to the open state. This finding explains why base flipping in base-pair kinetics could occur spontaneously in solution (Leijon and Graslund 1992; Snoussi and Leroy 2001). Interestingly, in our case, the base-flipping event requires the assistance of Exp5 to overcome the energy barrier from the low energy state (closed) (Fig. 7B, gray ribbon) to the high energy state (open) of the 2 -nt $3^{\prime}$ end (Fig. 7B, black ribbon).

To further understand the role of the 2-nt 3' overhang in pre-miRNA recognition by Exp5, we analyzed H-bonding networks between the $3^{\prime}$ overhang (G62 and C63) and the trap from the MD trajectory. Here, the criterion of the $\mathrm{H}$-bond is an $\mathrm{H}$-acceptor distance $<3.5 \AA$ and a donor-Hacceptor angle $>120^{\circ}$. The $\mathrm{H}$-bonding interactions listed in Table 1 show that G62 forms stable H-bonds through its phosphorous group with Arg598 during the whole simulation. C63 also produces several H-bonds mainly through its phosphorous oxygen with Arg835, Gln642, and Ser722. This reveals that the phosphate backbone of pre-miRNA, not specific bases, plays a pivotal role in $3^{\prime}$-overhang recognition, which is further demonstrated by the energy decomposition (for details, see "Energy Contribution Analysis" below).

To probe whether the overhang recognition is basedependent or not, we modified the 2-nt $3^{\prime}$ overhang $\left({ }^{62} \mathrm{GC}^{63}\right)$ with two different nucleotides based on the X-ray structure of Exp5-GTP-RNA (PDB code: 3A6P), since other

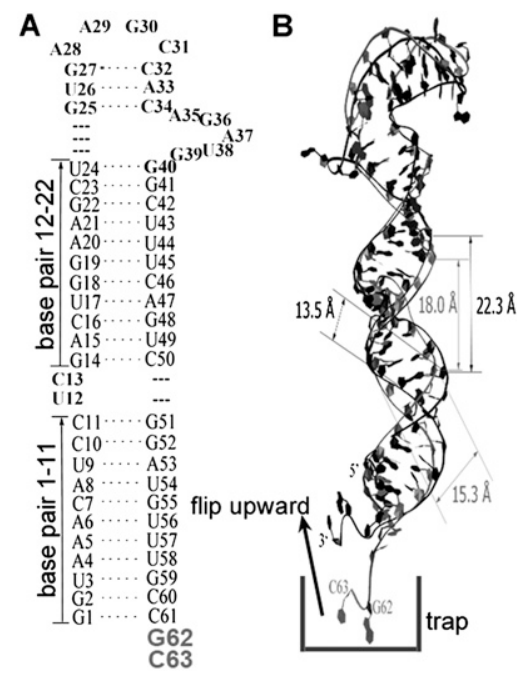

FIGURE 7. (A) Secondary structure of pre-miRNA30a (numbering is according to Okada et al. 2009). The missing bulged residues (U12 and C13) and hairpin loop residues (nucleotides 25-39) in the crystal structure are reconstructed by means of Sybyl. The 2-nt 3' overhang is highlighted in bold. (B) Conformation details of representative snapshots of free pre-miRNA along with the bound pre-miRNA. The major groove width (A8) of bound pre-miRNA is $4.3 \AA$ narrower than that of free pre-miRNA, while the minor groove width (A8) is 1.8 $\AA$ wider. A side view of the 2-nt $3^{\prime}$ overhang, with a flipping motion of these two bases, is shown. Both bases in free simulation point toward the minor groove. 
TABLE 1. H-bonding interactions between the 2-nt 3 ' overhang $\left({ }^{62} \mathrm{GC}^{63}\right)$ and the trap region

\begin{tabular}{llc}
\hline Donor & Acceptor & Occupancy $(\%)^{a}$ \\
\hline ARG602@NH & G62@O2P & 5.75 \\
ARG835@NH & C63@O1P & 10.03 \\
SER722@OG & C63@O3' & 10.46 \\
GLN642@N & C63@O1P & 12.79 \\
C63@N4 & GLU711@OE & 15.64 \\
ARG718@NH & C63@O & 15.73 \\
ARG835@NE & G62@O2' & 18.89 \\
ARG835@NH & G62@O2' & 26.62 \\
ARG598@NE & G62@O1P & 28.74 \\
ARG602@NH & G62@O1P & 32.58 \\
ARG718@NH & C63@N3 & 33.45 \\
ARG598@NH & G62@O1P & 43.26 \\
LY5830@N & G62@O6 & 50.61 \\
GLN642@N & C63@O2P & 52.55 \\
GLN642@NE2 & C63@O1P & 56.48 \\
ARG718@NE & C63@O & 60.16 \\
\hline
\end{tabular}

The percentage of simulation snapshots (saved every $10 \mathrm{psec}$ ) in which the $\mathrm{H}$-bond was present are listed.

${ }^{a}$ The occupancy of $\mathrm{H}$-bonds that are formed between the overhang and the trap larger than $5 \%$ is listed.

crystal structures of miRNA precursor are unavailable currently. Here, the mir-30 gene family (Homo sapiens) was selected as it has been widely investigated (Zeng et al. 2002; Zeng and Cullen 2004), ending up with a finding that there are only four different 2-nt $3^{\prime}$ overhangs $\left({ }^{62} \mathrm{UC}^{63}\right.$, ${ }^{62} \mathrm{CC}^{63},{ }^{62} \mathrm{CU}^{63}$, and ${ }^{62} \mathrm{GC}^{63}$ ) in the whole miRbase (http:// www.mirbase.org/), one of which, i.e., ${ }^{62} \mathrm{GC}^{63}$, has been simulated in this work. On this basis, we performed three additional simulations (10 nsec for each) for three other $3^{\prime}$ overhangs. Interestingly, the simulation results further demonstrate that it is the phosphate backbone, instead of the bases of pre-miRNA, that plays a key role in $3^{\prime}$-overhang recognition (Supplemental Tables S1-S3). Combined, all these suggest that the $3^{\prime}$-overhang recognition is in a sequence-independent manner, which theoretically proves why the base flipping is involved in the sequenceindependent recognition and exchange of sequence-degenerate single-stranded genetic material between bacteria (MacDonald et al. 2006).

Other than the flip-out mechanism used for recognition of the 2-nt 3' overhang by Exp5, the stem of the premiRNA is presumed to be recognized through a broad range of positively charged inner-surface residues of the Exp5-GTP binary complex (Okada et al. 2009). However, the formation of a higher-order structure usually requires that the conformation of the RNA template be bent or distorted to bring the requisite proteins into close proximity. Inspection of the X-ray structure of the Exp5-GTPRNA complex reveals that the protruded and kinked helix H9B closely interacts with the minor groove of pre-miRNA. Thus, some induced-fit effects may exist during the binding process of pre-miRNA. This, in turn, would facilitate the assembly and enhance the overall stability of the Exp5GTP-RNA complex. To support this idea, we also investigated the flexibility of pre-miRNA in both the free premiRNA and Exp5-GTP-RNA simulations. Accordingly, the helical parameters for the free pre-miRNA system as well as the bound conformation (Exp5-GTP-RNA) were calculated from the trajectories of average pre-miRNA structures using the program X3DNA (Lu and Olson 2003). We conducted the helicoidal analyses of the average structures from both trajectories as shown in Figure 8. Comparing the free state of pre-miRNA with its bound state, we observe some notable deviations, although the average structures appear to be close to canonical A. The pattern of bound pre-miRNA base-pair opening, as well as the propeller, is almost reciprocal to that of the free premiRNA, especially for the first $10 \mathrm{bp}$ (Fig. 8). Other helical parameters such as stretch and shear are remarkably in agreement between the two simulations. Furthermore, the groove parameters of the above two states of pre-miRNA were measured (Fig. 8). With respect to the free premiRNA, the minor-groove widths of bound pre-miRNA increased at the vicinity of base pairs from base pairs 6-8 and from base pairs 12-14 (Fig. 8), which are the most biologically significant part for pre-miRNA-Exp5 binding. The widening of these minor grooves is attributed to the wedge of certain protruding amino acids (Arg380 and Glu445, Asp1087 and Ala1091) that insert into the minor groove of pre-miRNA. For instance, the minor-groove widths of the bound pre-miRNA widened from 15.0 to $15.6 \AA$ at A8/U54 (base pair 8) and from 14.8 to $15.8 \AA$ at A15/U49 (base pair 13) (Fig. 8). Since the widened minor grooves from base pairs 6-8 and from base pairs 12-14 naturally squeeze the central part, the width of a minor groove around the central part (base pair 10) has largely decreased in the RNA bound state. In addition, the bulge

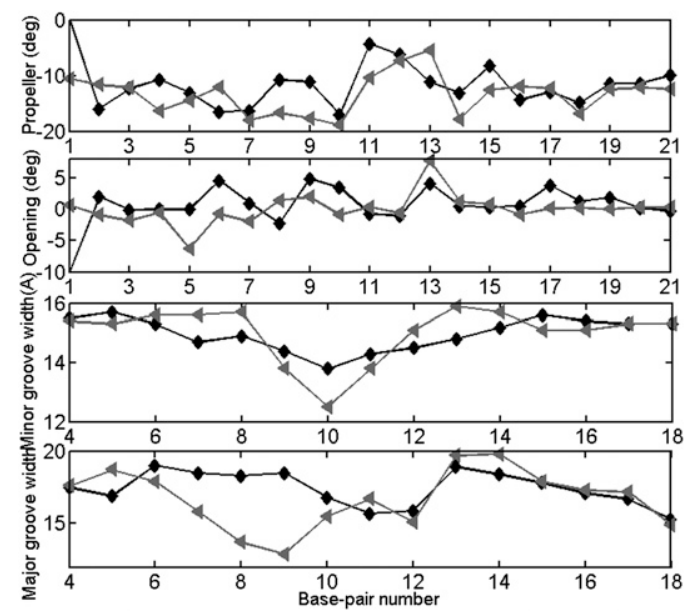

FIGURE 8. Helical base-pair parameters of time-averaged structures of free pre-miRNA (diamond) and bound pre-miRNA (left-triangle). The base-pair number is the same as in Figure 7A. 
dinucleotide $\left({ }^{12} U C^{13}\right)$ (Fig. 7) in the central stem of pre-miRNA shows some impacts on the minor groove width between flanking base pairs (base pairs 11 and 12 in Fig. 8).

For the major grooves, the overall widths of bound pre-miRNA are larger than those of free pre-miRNA. An interesting observation is made for base pairs $6-10$ of the free pre-miRNA. It adopts an unusually widened major groove conformation reminiscent of that in the $\mathrm{A}^{\prime}$-form RNA, which makes the basic patch (residues Lys132 to Lys141) of RanGTP easily access pre-miRNA. Once a pre-miRNA binds to RanGTP, the insertion of Lys132 and Lys134 (on the basic patch) into pre-miRNA (Supplemental Fig. S7) narrows the major groove of base pairs 7 and 8 and thereby widens the neighboring minor grooves contacting with the $\alpha$-helix H9B of Exp5. Consequently, the pre-miRNA forms the shape of a relatively stenosed major groove, which is even narrower than their minor-groove widths (base pairs 7-9), reminiscent of those in A-form RNA (Fig. 7). Taken together, these findings suggest that the changes of helical conformation in bound pre-miRNA (especially for 6$10 \mathrm{bp}$ adopting an A-form-like conformation) modulate the structural complementarities between the interacting protein and target pre-miRNA molecules, thus facilitating the assembly of the Exp5-GTP-RNA complex.

\section{Trap rearrangement is relevant to the binding of 3' overhang}

The 2-nt 3' overhang of pre-miRNA could fall into a socalled trap of Exp5. However, the dynamic recognition between pre-miRNA and Exp5 during this process remains unclear. To address these, we have superimposed the timeaveraged structures (last $15 \mathrm{nsec}$ ) of Exp5 extracted from apo-Exp5, Exp5-GTP, and Exp5-GTP-RNA (Fig. 9I). In the apo system, we observe a small trap with an entrance area of $\sim 165 \AA^{2}$ (cross-section), which cannot accommodate the overhang of pre-miRNA due to its much bigger area $\left(\sim 190 \AA^{2}\right)$. This is further evidenced by the strong steric clash between the $3^{\prime}$ overhang (G62) (blue in Fig. 9) of pre-miRNA and the I12 (magenta in Fig. 9) of apo-Exp5 (blue grid in Fig. 9II). While for the binary system, this trap entrance is enlarged significantly to $\sim 256 \AA^{2}$ with the binding of RanGTP (Fig. 9III), resulting in the disappearance of steric hindrance between I12 (yellow in Fig. 9) in binary model and G62 (blue in Fig. 9) in the ternary model (Fig. 9II). Notably, after the uptake of the 2-nt 3' overhang of pre-miRNA, the trap collapses again with the entrance area of $202 \AA^{2}$, which is discussed in more detail in this section.

\section{apo-Exp5 versus Exp5-GTP}

Figure 10A-I shows the superposition of apo-Exp5 and Exp5-GTP. For the apo system, we observe that the I12 exhibits high stability over the entire MD trajectory (RMSF = $\sim 1.2 \AA)$, sealing the entrance of the trap like a big plug (magenta cartoon in Fig. 10A-II). Further analysis shows that I12 forms three H-bonds between Glu588, Lys830 (in the "Long loop" in Fig. 1), and Arg598 (in H12B), which strongly stabilizes this loop. After binding of RanGTP, the H-bond networks between the I12 and the "Long loop" are broken, and I12 moves to the I10 position, thus opening the trap entrance (yellow cartoon in Fig. 10A-II). In addition, the two sides of Exp5 (H1-H9 and H15-H20) (Figs. 1, 10A) shift slightly upward and shrink inward relative to the apo model (yellow arrows in Fig. 10A-I). The systematic movement of the protein makes I10 (a loop connecting H10A and $\mathrm{H} 10 \mathrm{~B}$, residues 471-498) transit from a compact to an extended loop, which is further H-bonded to I12 (between Ser491 [HG] and Pro592 [O], Thr487 [H] and Lys590 [O]), thus stabilizing the enlarged trap (Fig. 10A-II).

\section{Exp5-GTP versus Exp5-GTP-RNA}

The superposition of Exp5-GTP with Exp5-GTP-RNA illustrated in Figure 10B-I shows that after the binding of 


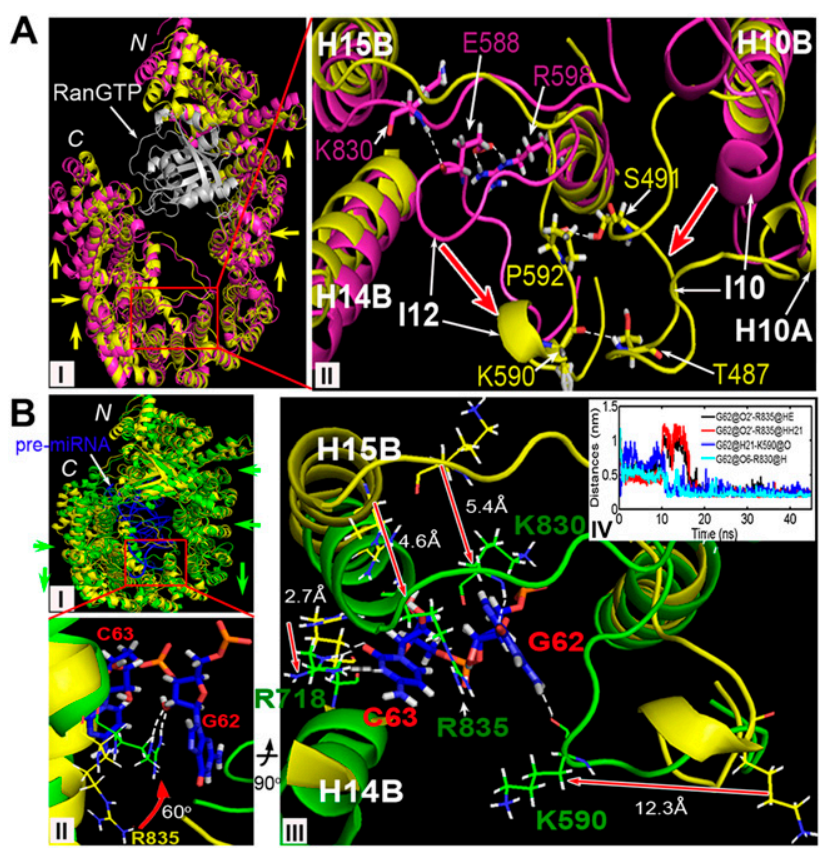

FIGURE 10. (A-I) Structural superposition of apo-Exp5 (magenta) and Exp5-GTP (yellow). Residues are displayed in stick representation, and their atoms are colored as follows: (white) hydrogens; (magenta) carbons in apo-Exp5; (yellow) carbons in binary complex; (blue) nitrogen; (red) oxygens. (A-II) The projection shows the conformational changes of the trap in the two models; (red arrows) the movement directions of the two loops (I10 and I12) in binary complex. (B-I) Structural superposition of Exp5-GTP (yellow) and Exp5-GTP-RNA (green). (B-II) Top view of the trap (from mouth to bottom) showing the rotation movement of Arg835 (red arrow). For clarity, only Arg835, G62, and C63 are shown. The Arg835 $\mathrm{NH}_{2}$ group donates two H-bonds to $\mathrm{O}^{\prime}$ ' of $\mathrm{G} 62$, separating the trap into two subsections to hold C63 and G62, respectively. (B-III) The projection highlights the side view of the trap, showing the downward translation of Lys590, Arg718, Lys830, and Arg835 (line representation) in ternary complex. (B-IV) Hydrogen-bond lengths of G62O2'-Arg835HE (black), G62O2'-Arg835HH21 (red), G62H21-Lys590O (blue), and G62O6-Arg830H (cyan) in ternary complex in MD trajectory.

pre-miRNA, the two sides of Exp5 shrink inward and tightly clamp pre-miRNA (green arrows in Fig. 10B-I), which is consistent with the radius of gyration analysis (Fig. 3). Particularly, the downward movements of H14B, H15B, and the "Long loop" (Fig. 9II), in conjunction with the leftward movement of I12, result in a large displacement of residues around the trap entrance, thus shrinking the trap. Further analysis shows that there are several key residues for stabilizing the $3^{\prime}$ overhang of pre-miRNA, i.e., Lys590, Arg718, Lys830, and Arg835 around the trap (Fig. 10B-II, B-IV). Compared with the Exp5-GTP system, the downward translation of the guanidinium group of $\operatorname{Arg} 718(\sim 2.7 \AA)$ and the amino group of Lys830 $(\sim 5.4 \AA)$, together with the leftward movement of Lys590 $(\sim 12.3 \AA)$, successfully hook the bases of the $3^{\prime}$ overhang. It is quite striking that the backbone of Arg835 moves downward $\sim 4.6 \AA$, and its side chain rotates downward $\sim 60^{\circ}$ through the CD-CG bond, whereby the $\operatorname{Arg} 835 \mathrm{NH}_{2}$ group donates two $\mathrm{H}$-bonds to
O2' of G62 with the H-bond length of $\sim 2.3 \AA$ (Fig. 10BIII). These two H-bonds operate as a "partition wall," making the trap convert from a "single" to a "duplex" family dwelling that contains two dwelling units for accommodating C63 and G62, respectively.

In view of the aforementioned conformational changes in the three Exp5 structures, an unexpected mechanistic insight emerges that the H-bonds between $\mathrm{I} 12$ and the "Long loop" can lock the entrance of the trap to prevent the insertion of the 2-nt 3' overhang into it, thereby preventing the rebinding of pre-miRNA after its dissociation. The binding of Ran can relieve the topological stress of the trap, paving the way for accommodation of the $3^{\prime}$ overhang of pre-miRNA.

\section{Energy contribution analysis}

To better understand how pre-miRNA is recognized by Exp5, a free-energy decomposition scheme was developed to determine binding energy hot spots of the Exp5-GTP-RNA complex. The binding free energy per residue is presented in Supplemental Table S4. The results show that the two ends and the central stem of pre-miRNA are the primary energy contributors (Fig. 11), as these regions form strong $\mathrm{H}$-bonds with Exp5. Especially, G1, C61, G62, and C63 extensively interact with the tunnel of Exp5 with a high relative contribution ratio $(>10 \%)$ to total binding energy, which further reveals that the terminal part of pre-miRNA plays an critical role in Exp5 recruitment of RNA (Fig. 11, gray stems). On the contrary, the energy contribution of the loop nucleotides is distinct from those of the two ends and the central stem of pre-miRNA, making negligible contributions. Decomposition of energy into backbone and side-chain atoms reveals that the favorable contribution of most nucleotides, such as G62 and C63 ( $3^{\prime}$ end), arises essentially from their backbone phosphate groups. These results are in good agreement with the above analysis (see "Exp5 Recognizes the pre-miRNA through the Base Flipping of the 2-nt 3' Overhang and Widening of the Minor Groove of the Stem" above). For examples, G1, C61, and G62 form H-bonds to Exp5 mainly through phosphate groups (from Table 1), and so do G48 and U49 with Ran (Lys132 and Lys134).

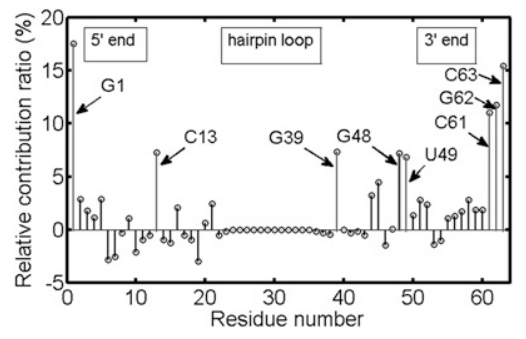

FIGURE 11. Relative contribution ratio of each residue of premiRNA to the binding free energy. The residue number is the same as in Figure 7A. The eight dominant residues are labeled. 


\section{RanGTP decreases the dissociation barrier for pre-miRNA}

From the above section "Energy Contribution Analysis," we have found that upon the binding of RanGTP, the binding surfaces between pre-miRNA and free Exp5 showed a higher degree of shape complementarity with remarkably high binding energies (Okada et al. 2009), indicating that pre-miRNA is apt to be assembled in the presence of RanGTP. This raises the question whether the existence of Ran also facilitates the dissociation of premiRNA from Exp5 or not? To address this, we have carried out SMD simulations by applying external constant forces on the pre-miRNA to "pull apart" the complex. The results show that the pre-miRNA in both systems can withstand two small external constant forces $(1000$ and $2000 \mathrm{~kJ} / \mathrm{mol}$ per $\AA^{2}$ ) before breaking apart. And when the force increases to 10,000 and $11,000 \mathrm{~kJ} / \mathrm{mol}$ per $\AA^{2}$, respectively, the Exp5GTP-RNA complex structure but not the Exp5-RNA complex starts to decompose. Taking the result of the $10,000 \mathrm{~kJ} / \mathrm{mol}$ per $\AA^{2}$ external force as an example, for the ternary structure after $3.5 \mathrm{nsec}$, we observe a sharp increase (>6 $\mathrm{A} / \mathrm{nsec}$ ) in the stretch distance, indicating that this complex is being opened in response to the large pull force (Fig. 12A, gray curve). For the binary structure, however, at the first $4.5 \mathrm{nsec}$, its stretch distance shows a slow decrease (Fig. 12A, black curve), which indicates that in the absence of RanGTP, the binding of pre-miRNA to Exp5 exhibits a stronger resistance against external decompounded forces. The similar results of the $11,000 \mathrm{~kJ} / \mathrm{mol}$ per $\AA^{2}$ external force are shown in Supplemental Figure S8.

Here we encounter an apparent paradox: The above results of SMD simulations have shown that the binding of pre-miRNA is more stable in the absence of GTP. However,

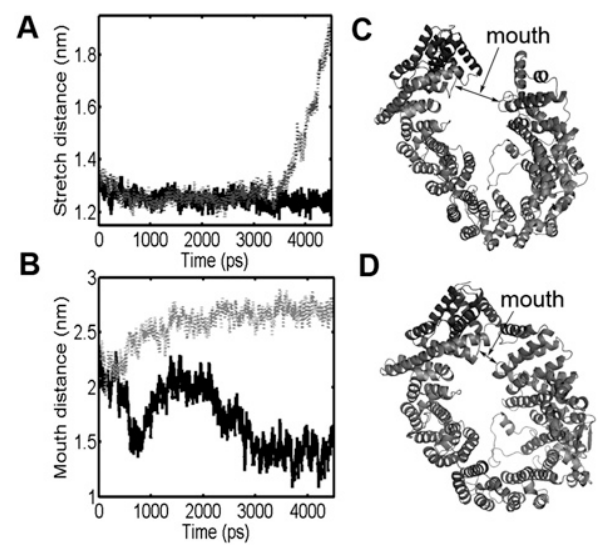

FIGURE 12. (A) Time evolution of the stretch distances between premiRNA and Exp5 during the Exp5-RNA (gray) and Exp5-GTP-RNA (black) complex simulations. (B) Corresponding time evolution of mouth distances of Exp5 in binary (gray) and ternary (black) states. Two representative structures of Exp5 are extracted from $(C)$ ternary and $(D)$ binary simulations. The mouth distance in ternary simulation $(C)$ is significantly larger than that in binary simulation $(D)$. the analysis of $\mathrm{H}$-bonds between the basic patch of RanGTP, especially for the fork residues Lys132 and Lys134 and the phosphate groups of C7, A8, G48, U49, and $\mathrm{C} 50$ of pre-miRNA reveals that there are on average four H-bonds between them during the whole trajectory, significantly contributing to their high-affinity binding $(\sim 28 \mathrm{~kJ} / \mathrm{mol}$ ) (Pace et al. 1996), which implies that the pre-miRNA should be more stable in the Exp5-GTP-RNA complex than in the Exp5-RNA complex.

To resolve this conflict, we examined the U-holder mouth distances (with the COMs of Gln168 and His1086 taken as reference) of the SMD trajectories. Prior to the stretching, both states of Exp5 have the same mouth distances with values of $\sim 2.4 \mathrm{~nm}$. Upon stretching, the mouth of Exp5 in the Exp5-RNA binary structure becomes smaller to $1.15 \mathrm{~nm}$ in width (Fig. 12B, black curve), causing the $\mathrm{N}$-terminal region $(\mathrm{H} 1-\mathrm{H} 8)$ comprised by many polar and positively charged residues, to tightly wrap the premiRNA stem (Fig. 12D) and consequently increase the binding affinity of pre-miRNA to Exp5. Conversely, the mouth of Exp5 in the Exp5-GTP-RNA structure becomes more open with the largest distance up to $\sim 2.9 \mathrm{~nm}$ (Fig. 12C; gray curve in Fig. 12B). Therefore, the potential interaction between the $\mathrm{N}$ terminus of Exp5 and premiRNA (as in the above binary system) is blocked by the Ran insertion. Compared with the $\mathrm{N}$ terminus of Exp5 binding to pre-miRNA, the contacts between Ran and premiRNA only mediated by the basic patch (particular for Lys132 and Lys134) of Ran and major grooves of premiRNA are very weak.

Overall, our SMD results, coupled with previous results, give insights into the pivotal role of Ran in assembly and dissociation of the ternary structure during the pre-miRNA export cycle. In the assembly process, RanGTP binding to Exp5 formed an overall shape complementarity that benefits pre-miRNA binding. In the dissociation process, in the presence of Ran, pre-miRNA appears to interact with the Lys132 and Lys134 of Ran instead of directly with the N-terminal regions of Exp5. Therefore, Ran's presence is required for pre-miRNA dissociation in the cytoplasm by decreasing the barrier for pre-miRNA dissociated from Exp5.

\section{GTP hydrolysis undermines the reassembly of Ran to Exp5 in cytoplasm}

Although the hydrolysis of GTP into GDP has been reported to be responsible for the significantly decreased binding affinity of Ran for Exp5 in the cytoplasm, which consequently leads to its release from Exp5 (Bohnsack et al. 2004), it is still poorly understood how the GTP hydrolysis decreases the binding affinity of Ran to Exp5 and thereby prevents the rebinding of Ran to Exp5. To explore the dynamical properties of Ran in the GTP state and the GDP state, we made a comparison between the Exp5-GTP-RNA and Exp5-GDP-RNA systems. 
To begin with, we investigated the hydrophobic contacts between Ran and Exp5 in both systems, which have been identified to play an important role in the stability of proteins (Phillips 2008), and found that the buried hydrophobic surface area of RanGTP complex is smaller $(\sim 100$ $\AA^{2}$ ) than that of RanGDP, which implies that RanGDP is more thermodynamically stable than RanGTP. By taking an average value for the enhancement of stability upon burial of the hydrophobic surface area (Vallone et al. 1998), we estimated a contribution of $\sim 7 \mathrm{~kJ} / \mathrm{mol}$ to thermodynamic stability in the RanGDP complex, and of $0 \mathrm{~kJ} / \mathrm{mol}$ to RanGTP.

Next, we turn our focus to the comparison of salt-bridge interactions. The analysis of salt bridges in Exp5-GTPRNA shows that four of the polar contacts are between residues on Helix S of Ran (Arg106, Asp107, and Gln113) and on Exp5 H3-H5 (Arg118 on H3B, Arg159 and Asp163 on H4B, and Arg222 on H5B) (Fig. 13A), which interlock $\mathrm{H} 3 \mathrm{~B}-\mathrm{H} 5 \mathrm{~B}$. Constrained by these salt bridges, the curvature of $\mathrm{H} 1-\mathrm{H} 9$ of Exp5 is stabilized (average RMSF $=1.3 \AA$ ), partly explaining why the stable core of Exp5 is predominantly formed by Ran and N-terminal regions of Exp5 (H1-H9), whereas for Exp5-GDP-RNA, these salt bridges between Ran and Exp5 are disrupted due to GTP hydrolysis (Fig. 13B). Since the GTP hydrolysis occurs by an in-line attack on the $\gamma$-phosphate by a water molecule, the interaction of Gly68... O $\gamma$ would be broken once GTP hydrolyzes, finally resulting in the relaxation of switch II (residues 65-80) (Fig. 14A). Along with the state transition of this switch, the residue Arg76 is translated rightward $\sim 1.5 \AA$ with its side chain rotating by $\sim 60^{\circ}$ and thereby

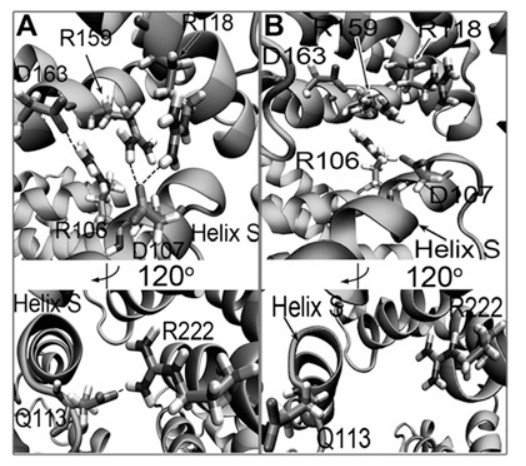

FIGURE 13. The comparison of salt-bridge interaction in Exp5GTP-RNA and Exp5-GDP-RNA systems. In all panels, backbones of Exp5 and Ran are shown in new-cartoon representations. Residues are displayed in licorice. (A) Salt bridges between Helix S of Ran (Arg106, Asp107, and Gln113) and Exp5 H3B-H5B (Arg118 on H3B, Arg159 and Asp163 on H4B, and Arg222 on H5B) in Exp5-GTP-RNA complex. (Top picture) The front view of the salt bridges between Helix S of Ran (Arg106 and Asp107) and H3B-H5B (Arg118 on H3B, Arg159 and Asp163 on H4B) of Exp5; (bottom picture) the back view of the salt bridges between Helix S of Ran (Gln113) and H3B-H5B of Exp5 (Arg222 on H5B). (B) These four salt bridges are disrupted in Exp5-GDP-RNA.

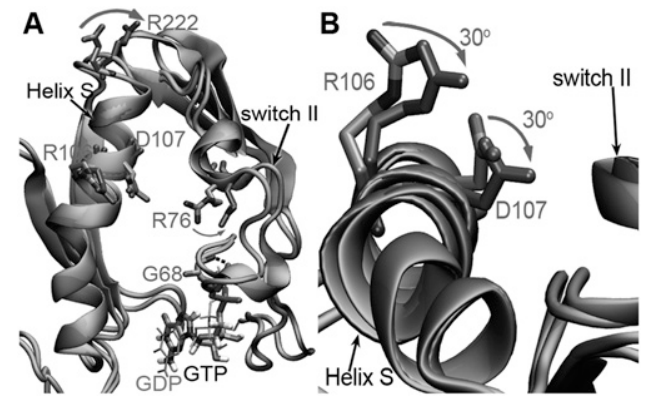

FIGURE 14. Structural superposition of time-averaged RanGTP and RanGDP, indicating that Helix S in Ran undergoes a rotation motion upon GTP hydrolysis. The residues in RanGTP conformation are shown in licorice representation. For a clear view, hydrogen atoms in the amino acids are removed. The residues in RanGDP conformation are shown in gray licorice representation. $(A)$ The switch II (residues 65-80) is relaxed in RanGDP (right) compared with that in RanGTP (left). Arg76 is translated rightward $\sim 1.5 \AA$, and its side chain rotates rightward by $\sim 60^{\circ}$ and thereby enlarges the space between the Helix $S$ and switch II. Gln113 located in the C terminus of Helix S breaks the salt bridge with Arg222 on H5B and moves toward the solvent. (B) The clockwise rotations of Arg106 and Asp107 to fill the enlarged space between Helix S and switch II are indicated by arrows.

enlarges the space between switch II and Helix S (Fig. 14A). Immediately, Helix $S$ in the RanGDP conformation rotates rightward to fill the enlarged space again due to the hydrophobic interactions between Ran and H1-H9 (Fig. $14 \mathrm{~B})$. This rotation is quantified by measuring the local rotation for all residues within the helix with respect to the position of the corresponding residue in the RanGTP conformation of the protein, defined from a local helical axis.

The rotation of Helix S forces its arginine (Arg106) and aspartic residues (Asp107) and Gln113 to cross this hydrophobic area. Hence, Arg106 and Asp107 (side chains) located in the Helix $S$ move toward switch II $\sim 30^{\circ}$ and break the corresponding salt bridges (Fig. 14B). Gln113 located in the $\mathrm{C}$ terminus of Helix $\mathrm{S}$ breaks the salt bridge with Arg222 on H5B and moves toward the solvent (Figs. $13 \mathrm{~B}, 14 \mathrm{~A})$. The binding affinity of Ran to Exp5 calculated based on these four salt bridges (average, $\sim 6 \mathrm{~kJ} / \mathrm{mol}$ each) (Schreiber and Fersht 1995) is $\sim 24 \mathrm{~kJ} / \mathrm{mol}$ in the RanGTP system, yet is zero in the RanGDP complex. Together with the affinity estimated according to the change of buried hydrophobic surfaces, a total of $17 \mathrm{~kJ} / \mathrm{mol}$ was gained for RanGTP, but 0 for RanGDP. In this view, Exp5 appears to have a significantly higher binding affinity for RanGTP than for RanGDP (Brownawell and Macara 2002), thus preventing RanGDP from rebinding to Exp5 in cytoplasm.

\section{DISCUSSION}

The free Exp5 structure is considered as the initial state at the beginning of the nuclear transport cycle. By atomistic MD simulations, we have observed a highly flexible and 
dynamical free Exp5 structure, which intrinsically undergoes large conformational changes and fluctuations. As indicated by PCA, with rotation and bending of $\mathrm{H} 1-\mathrm{H} 8$ and $\mathrm{H} 19 \mathrm{~B}-\mathrm{H} 20$ relative to $\mathrm{H} 9-\mathrm{H} 19 \mathrm{~A}$, free Exp5 experiences simultaneous opening motions in the nucleus. This is consistent with the recently determined crystal structure of Xpot in its unbound state, whose karyopherin superhelix is in an extended conformation (Cook et al. 2009). The opening of the $\mathrm{C}$ and $\mathrm{N}$ termini will evert the pre-miRNA binding sites to solution and thereby reduce its binding affinity. According to the RMSF analyses and conformation studies, residues in I12 lock the entrance of the trap to prevent the 2-nt $3^{\prime}$ overhang of pre-miRNA from falling into it. Thus, free in solution, the distorted inner surface of Exp5 and the stable I12 seal off the binding site to hinder pre-miRNA from accessing its receptor. This proposed geometry at least partly explains why pre-miRNA cannot bind to free Exp5 without association of RanGTP in the nucleus (Zeng and Cullen 2004). In its free state, Exp5 experiences a transition from a compact to an extended state, resulting in the primary binding sites accessible for RanGTP. Interestingly, our water density analysis also showed that the solvent density is low in the Ran binding sites, revealing the fact that hydrophobic forces are important for RanGTP binding to Exp5 (Fig. 15A, left; Phillips 2008).

As the transport cycle begins, free Exp5 bound with RanGTP forms a stable binary complex with a tight curvature (Fig. 15A, center), thus connecting the mouth of the extremely flexible Exp5. This step comprises the bending and kinking of several A helices of HEAT repeats and many minor rearrangements in inter-HEAT repeat angles. Exp5 rigidified by RanGTP restores energy to compensate for distortions introduced into Exp5 by complex formation or compensates for the loss of entropy resulting from its reduced flexibility. This mechanical energy stored by distorting the molecules may be used to balance the large interaction energy deriving from extensive interaction interfaces to enable transport complexes to be disassembled by relatively small energy changes (Conti et al. 2006).

Our results show that RanGTP binding to Exp5 forms an overall shape complementarity benefiting pre-miRNA binding. This raises concern about what kind of conformational change of pre-miRNA facilitates its recognition by Exp5. By examining the conformational dynamics of premiRNAs, we observed a stable structure of the free premiRNA in solution with the 2-nt $3^{\prime}$ overhang adopting a fully closed state, which possesses a more compact $3^{\prime}$ end to reduce the steric barriers during its binding to Exp5. As the terminal nucleotides of pre-miRNA approach the bottom of Exp5, the exposed and negatively charged phosphodiester groups of the $3^{\prime}$ end begin to flip down to an extended state with the assistance of Exp5 and are easily captured by the trap region, thus initializing construction of the complex. In such a 2 -nt 3 '-overhang

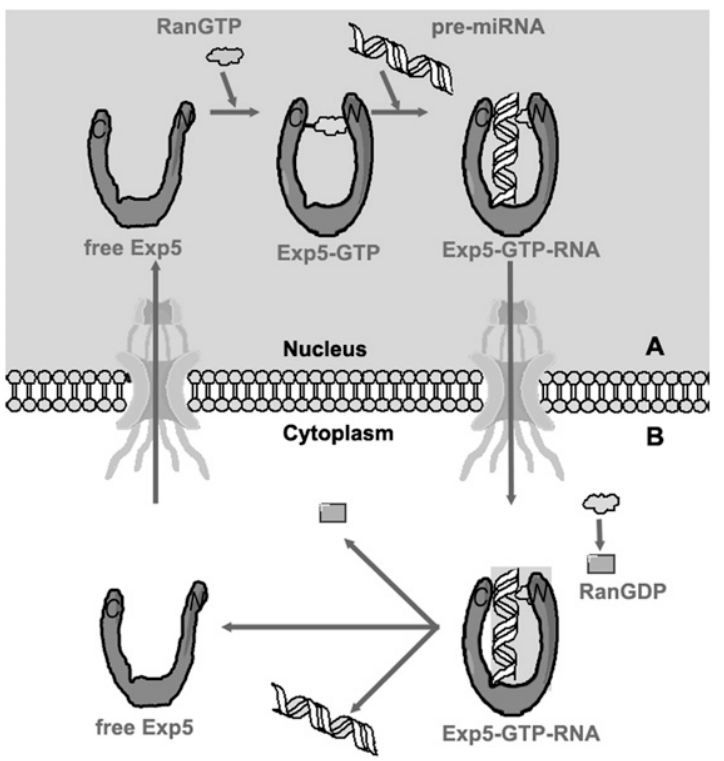

FIGURE 15. Schematic diagram of RanGTP-assisted pre-miRNA binding to Exp5 and protein dynamics in the Nuclear Export Cycle. $(A$, left $)$ Free Exp5 adopts an open cytosolic conformation in solution. (A, center) RanGTP binds to the extended Exp5 through its switch I, switch II, and basic patch and forces Exp5 to form a U-like holder facilitating pre-miRNA binding. ( $A$, right) The flipping-out motion of the 2-nt 3' overhang and the indirect readout mechanism allow premiRNA to be rapidly recognized by Exp5. The formed export complex is translocated through the NPC. ( $B$, right) Due to RanBP1 binding, RanGTP and pre-miRNA are released from Exp5, then RanGTP hydrolyzes to RanGDP, which has a very low binding affinity for Exp5. ( $B$, left) Dissociation releases the constrained Exp5, which distorts and exposes its basic inner surface to the cytoplasm, preventing pre-miRNA from rebinding.

recognition process, we have identified phosphodiester groups as the major contributors instead of bases, demonstrating why this process proceeds in a sequence-independent manner. This is consistent with the fact that different pre-miRNAs even with no consensus sequences can all be recognized and exported by Exp5.

It is reasonable to expect that protein-pre-miRNA association would be facilitated by enhanced ease of RNA deformation and a switch between intrinsic RNA shape in solution and the strained RNA conformation in the complex. Our data suggest that the recognition of the pre-miRNA stem by Exp5 (partially at least) relies on an indirect readout mechanism that can be mediated by means of changes in structural parameters such as the propeller and opening. In addition, according to groove parameters analysis, it is found that the pre-miRNA stem with two double-stranded RNA helices adopts an unusual widened minor groove conformation reminiscent of those in A'-form RNA (Fig. 7B). Very recently, Bullock et al. (2010) have identified the Drosophila melanogaster $f_{s}(1)$ K10 transcripts, which form a stem-loop with two double-stranded RNA helices and adopt an unusual $\mathrm{A}^{\prime}$-form conformation with widened major grooves reminiscent of those in 
B-form DNA. With structure determination of four mutant RNAs and extensive functional assays in Drosophila embryos, the $\mathrm{A}^{\prime}$-form RNA helices have been found as critical recognition sites for cytoplasmic mRNA transport. Thus, it is reasonable to suggest that the mechanism of local transition from $\mathrm{A}^{\prime}$-form to A-form RNA helices also probably represents a recognition site during the pre-miRNA nuclear export. When binding to Exp5, pre-miRNA uses the local transition mechanism to modulate the structural complementarities between the interacting protein and target premiRNA molecules, thus facilitating the assembly of the Exp5-GTP-RNA complex (Fig. 15A, right). Furthermore, it is worth noticing that phosphate groups of C7, A8, G48, $\mathrm{U} 49$, and C50 of a pre-miRNA major groove form $\mathrm{H}$-bonds to the basic patch of Ran. These H-bond interactions contribute to the main affinity for the pre-miRNA binding to RanGTP, enhancing the stabilization of the Exp5-GTPRNA ternary structure. Combined, these results extend the previously biochemical study that complex formation with RanGTP greatly enhanced the affinities of most substratespecific exportins for export cargos (Fried and Kutay 2003), and add atomic details.

After passing through the pores to the cytosol, RanGTP hydrolysis, which must be stimulated by RanGAP, does not occur directly on the transport receptor-bound RanGTP but rather on the RanGTP dissociated from the exportin by help of RanGTP binding proteins of the RanBP1 family (Bischoff and Görlich 1997). This process is required for unloading the cargo (Fig. 15B, right). A combination of simulations of the Exp5-GDP-RNA and Exp5-GTP-RNA complexes reveals that the four salt bridges $(\sim 24 \mathrm{~kJ} / \mathrm{mol}$ in total) are broken upon GTP hydrolysis, reducing the binding affinity of Ran for Exp5 considerably. This result is consistent with the biochemical study that Exp5 has high affinity for RanGTP in the nucleus and very low affinity for RanGDP in the cytoplasm (Brownawell and Macara 2002), and adds atomic detail, suggesting that GTP hydrolysis hampers Ran reassembling to Exp5.

During the substrate dissociation process, SMD simulations are used to investigate the unbinding events, which are based on applying a guiding force to a system with the purpose of moving the system over barriers in a time frame that is normally inaccessible, i.e., too long, without the extra force. Usually, the pulling direction of the spring in SMD is chosen randomly or by guessing on the basis of the structural information, thus the force applied to the ligand in such chosen directions may not move it along a favorable pathway. In the present study, to overcome this limitation, we have performed a series of SMD simulations with different directions of pulling force prior to the actual simulations to identify the possible directions. Finally, we chose the direction of force to be parallel to the RNA axis because this allows for relatively few possible bumps with the protein when the ligand is pulled out. The SMD studies agree with the observation made on the normal MD simulation that Exp5 closes its mouth in the Exp5-RNA complex (data not shown), providing a strong support for the idea that the removal of Ran will increase the barrier for pre-miRNA dissociated from Exp5. Thus, we reasonably speculate that the RanGTP dissociation with the assistance of RanBP1 occurs simultaneously with or lags behind the release of pre-miRNA from Exp5 (Fig. 15B, left). This is different from the proposal that the Ran dissociation triggers exportins shifting equilibrium toward the inactive conformation and decreases the substrate affinity, and then substrates are released into the cytoplasm (Ossareh-Nazari et al. 1997; Stade et al. 1997).

Last, we show that the dissociation of Ran and cargo makes Exp5 into an extended U-like state, and this open conformation of the unbound state distorts the cargobinding surface. This geometry, which has low binding affinity for RanGDP, is consistent with its function in preventing the pre-miRNA from rebinding to avoid their aggregation. The mechanism ensures that Exp5 mediates a high-performance and one-way transition. In contrast to the fact that Exp5 undergoes a transition to an extended state after dissociation of its binding partners, the exportin CAS/Cselp is more compact in the unbound state than in the ternary export complex. It is suggested that the presence of an intra-molecular interaction, which constrains the karyopherin superhelix in a tight ring-shape structure, seals off the cargo-binding sites, keeping cargo from rebinding to CAS/Cselp in the cytosol (Cook et al. 2005; Zachariae and Grubmüller 2006).

\section{CONCLUSION}

Here, we have investigated the structure and dynamics of the free state of Exp5 in solution and the mechanisms underlying its interactions with RanGTP/RanGDP and cargo by MD simulations, revealing both the nuclear and cytosolic snapshots of this transport factor. Our main findings are summarized as follows:

1. Our MD simulations and PCA analyses reveal a striking transition of Exp5 upon dissociation of RanGTP and pre-miRNA. Basically, free Exp5 is divided into two segments, $\mathrm{H} 1-\mathrm{H} 8$ and $\mathrm{H} 9-\mathrm{H} 20$. The dynamic mode of free $\operatorname{Exp} 5$ is related to RanGTP connecting the flexible $\mathrm{N}$ and $\mathrm{C}$ termini of Exp5 to form an overall shape complementarity, paving the way for pre-miRNA binding.

2. More water molecules are located at the inner surface of the protein, particularly at the trap region; while fewer water molecules reside on the outer surface and RanGTP binding sites. The highly asymmetric and anisotropic water distribution observed around the protein surface is functionally relevant.

3. Pre-miRNA binds to Exp5 via a flipping motion of the 2 -nt 3 ' overhang and the widening of the minor groove 
of the stem. For $3^{\prime}$-overhang recognition, the $\mathrm{H}$-bonds between non-specific phosphorous oxygen molecules of pre-miRNA and Exp5 trap residues show why this recognition appears in a sequence-independent manner to form an RNA-protein complex. For stem recognition, the mechanism of transition from $\mathrm{A}^{\prime}$-form to A-form RNA helices is required.

4. RanGTP does not merely cooperate with the premiRNA binding by altering the geometry of Exp5 to accommodate pre-miRNA, but also controls pre-miRNA binding by $\mathrm{H}$-bonding to pre-miRNA. In addition, existence of Ran can ensure Exp5 an appropriate structural geometry for pre-miRNA dismounting from the complex. Therefore, it is more favorable for RanGTP dissociation at the same time or after the release of premiRNA from Exp5.

Overall, our study provides an in-depth analysis of the nuclear export process of pre-miRNAs, especially on the coordination of interactions and the basis for molecular recognition and dissociation that underlies the process. This will be important for a full understanding of the export mechanisms and may also be relevant to other nuclear export studies.

\section{MATERIALS AND METHODS}

\section{Protein reconstruction}

The initial configuration was the ternary structure labeled $A$ in the asymmetric unit from the crystal structure of the Exp5-RanGTPpremiRNA (named Exp5-GTP-RNA for short) complex (PDB code: 3A6P, resolution $2.9 \AA$ ) (Okada et al. 2009). In that complex, several residues (residues 1, 474-490, 705, 706, 938951, 980-1009, and 1137-1204 for Exp5; residues 1-6 and 177216 for Ran; nucleotides 12, 13, and 25-39 for pre-miRNA) are missing. Using the crystal structure of Exp5-GTP-RNA as a starting structure for our simulations required re-creating the loop or helix between HEAT repeats in Exp5 and the loop or mismatched regions of the RNA that are missing in the crystal structure. The other missing terminal residues (residues 1 and 1137-1204 of Exp5, and residues 1-6 and 177-216 of Ran) were not reconstructed. The missing hydrogen atoms, main chains, and side chains in the crystal structure were reconstructed by using the Biopolymer module in the Sybyl 6.9 package (Tripos Associates). The spatial environment of each new residue was checked for close contacts or overlaps with neighboring residues, and stereochemical regularization of the structures was obtained by the Powell minimization method implemented in the Sybyl program. Before running simulations, protonation states of titratable groups of Exp5 were determined by using Whatif (Vriend 1990). In addition to the reconstructed Exp5-GTP-RNA system described above, we also constructed five other systems: Exp5GDP-RNA, Exp5-GTP, Exp5-RNA, apo-Exp5, and free premiRNA. The Exp5-GDP-RNA structure was built by replacing GTP with GDP in the Exp5-GTP-RNA complex, and Exp5-GTP was obtained by removing pre-miRNA from the ternary system. The deletion of RanGTP produces Exp5-RNA. apo-Exp5 and free pre-miRNA were directly extracted from the Exp5-GTP-RNA complex.

\section{Molecular dynamics protocol}

All MD simulations (Table 2) were performed with the GROMACS 4.0.4 package (Hess et al. 2008), using the amber03 force field (Duan et al. 2003). The polyphosphate parameters for GTP and GDP were collected from the work of Meagher et al. (2003). All systems were solvated in a rectangular box of TIP3P water (Jorgensen et al. 1983), keeping a minimum distance of $12 \AA$ between the solute and each face of the box. Each box was neutralized, and $0.15 \mathrm{M}$ sodium chloride was added to each simulation box. All solvated models were then optimized (5000 steps of steepest descent and 10,000 steps of conjugate gradient minimizations) to relax any steric conflicts generated during the setup. The solvent molecules in the minimized models were then heated up to $300 \mathrm{~K}$ and equilibrated for $200 \mathrm{psec}$ with the positional restraints on the protein heavy atoms by using a force constant of $k=1000 \mathrm{~kJ} / \mathrm{mol}$ per $\mathrm{nm}^{2}$. The MD simulations were performed in the NTP ensemble at constant pressure (1 atm) with isotropic position scaling and at $300 \mathrm{~K}$ with the Berendsen temperature coupling $T_{\mathrm{p}}=2 \mathrm{psec}$. The pressure was coupled to a Berendsen barostat with $T_{\mathrm{p}}=4 \mathrm{psec}$ and an isotropic compressibility of $4.5 \times 10^{-5} \mathrm{bar}^{-1}$ in the $x, y$, and $z$ directions (Berendsen et al. 1984). The integration time step was $2 \mathrm{fsec}$ with all bonds constrained according to the LINCS algorithm (Hess et al. 1997).

The short-range van der Waals energy was truncated at $10 \AA$. Electrostatic interactions were calculated explicitly at a distance smaller than $10 \AA$. To achieve an accurate and efficient treatment of the long-range electrostatic interactions, the smooth particlemesh Ewald (PME) method (Darden et al. 1993) was performed, with a grid spacing of $0.12 \mathrm{~nm}$. Finally, up to $45-\mathrm{nsec}$ long production runs for each of the systems and trajectory data were generated by free (unbiased) MD simulations. Considering the large conformational rearrangement of isolated pre-miRNA and Exp5, tackling the issue of incomplete sampling is particularly
TABLE 2. Overview of MD simulations performed for different simulations

\begin{tabular}{llcc}
\hline Systems & \multicolumn{1}{c}{ Description } & Charge & Simulation time \\
\hline Exp5-GTP-RNA & Exp5 + RanGTP + pre-miRNA & -83 & $45 \mathrm{nsec}$ \\
Exp5-GDP-RNA & Exp5 + RanGDP + pre-miRNA & -82 & $45 \mathrm{nsec}$ \\
Exp5-GTP & Exp5 + RanGTP & -20 & $45 \mathrm{nsec}$ \\
Exp5-RNA & Exp5 + pre-miRNA & -92 & $45 \mathrm{nsec}$ \\
apo-Exp5 & Exp5 & -29 & $45 \mathrm{nsec}$ \\
apo-Exp5 (R1) & The first replica of apo-Exp5 system & -29 & $45 \mathrm{nsec}$ \\
apo-Exp5 (R2) & The second replica of apo-Exp5 system & -29 & $45 \mathrm{nsec}$ \\
pre-miRNA & Single pre-miRNA & -63 & $45 \mathrm{nsec}$ \\
pre-miRNA (R1) & The first replica of pre-miRNA system & -63 & $45 \mathrm{nsec}$ \\
pre-miRNA (R2) & The second replica of pre-miRNA system & -63 & $45 \mathrm{nsec}$ \\
\hline
\end{tabular}


germane in this study. Thus, we performed the combination of significantly longer trajectories and the multicopy approach (Caves et al. 1998; Ivetac and McCammon 2009) for both systems. This combination allows for obtaining a more exhaustive conformational sampling compared to individual, shorter trajectories. The method has been described in detail by Caves et al. (1998). Here, we present only a brief description of essential features of the approach. A separate equilibration (and subsequent production run) was performed for each of the two extra replicas of each system by using a different randomization seed (a different positive integer) for the initial atomic velocities (Caves et al. 1998; Ivetac and McCammon 2009). The initial $10 \mathrm{psec}$ of the simulation was used as a temperature-control phase. After the original velocity assignment at $300 \mathrm{~K}$, velocities were reassigned every $0.2 \mathrm{psec}$ for the first $2 \mathrm{psec}$. Subsequently, the temperature was monitored every 0.2 psec for $2 \mathrm{psec}$, and if it is out of range of $300 \pm 5 \mathrm{~K}$, the velocities were scaled uniformly to $300 \mathrm{~K}$. This scaling procedure was performed every 0.4 psec for the next 2 psec. Then the velocities were kept constant so that the total energy was conserved. All replicas were extended to $45 \mathrm{nsec}$. For analysis, the three 45-nsec trajectories from each system were concatenated into a single 135-nsec trajectory (Caves et al. 1998; Ivetac and McCammon 2009). The coordinates of the simulated complexes were written out every 10 psec for subsequent analyses.

\section{PCA}

In this study, the RMSD differences between individual copies of the same simulation system suggest that the multicopy approach has been successful in generating more conformational variations than a single trajectory (Supplemental Fig. S5). To distinguish the functionally relevant collective motions from local "noise," an important method that has been used is the "combined essential dynamics" approach, whereby PCA is performed on the concatenated MD trajectory (van Aalten et al. 1995a,b; Ivetac and McCammon 2009). Especially in large complex systems, this approach plays a vital role in extracting the most important information that pertains to the essential physics of a biomolecular process such as protein assembling or molecular recognition.

PCA allows the trajectory obtained from MD simulations to be analyzed by reducing the degrees of freedom of the system to lower dimensions. For simplicity, we only considered the coordinates of a number $N$ of $\mathrm{C}_{\alpha}$ atoms since these subsets capture most of the conformational changes in the protein (Amadei et al. 1993). After eliminating the overall translational and rotational motion from the MD simulation through least-squares fitting, a positional covariance matrix was first constructed based on the three-dimensional (3D) positional fluctuations of $\mathrm{C}_{\alpha}$ atoms from their ensemble average position.

$C_{i j} \equiv\left\langle\Delta x_{i} \Delta x_{j}\right\rangle \equiv\left\langle\left(x_{i}-\left\langle x_{i}\right\rangle\right)\left(x_{j}-\left\langle x_{j}\right\rangle\right)\right\rangle,,(i, j=1,2,3, \ldots, 3 N)$

where brackets denote an average over the time frames in the simulation, $x_{i}$ is a Cartesian coordinate of the $i$-th $\mathrm{C}_{\alpha}$ atom, and $N$ is the number of $\mathrm{C}_{\alpha}$ atoms considered.

Then the eigenvectors and corresponding eigenvalues, which represent the direction and amplitude of the motion, respectively, were identified by diagonalizing the covariance matrix. The eigenvectors are ranked by the size of their corresponding eigenvalues, the "first" eigenvector being the one with the largest eigenvalue. Generally, the overall internal motion of the protein can be adequately described using only a few ("essential") eigenvectors with large eigenvalues. The central hypothesis of this method is that the more biologically significant motions would correspond to those eigenvectors with the larger eigenvalues. For visualization of the motions represented by the eigenvectors, the structures from the ensembles can be projected onto each eigenvector of interest and transformed back into Cartesian coordinates. The identification of dynamic domains and hinge regions based on the PCA results was performed using the DYNDOM program (Hayward and Berendsen 1998).

\section{Free energy decomposition}

The binding free energies were calculated using the Molecular Mechanics Poisson Boltzmann Surface Area (MM-PBSA)/Molecular Mechanics Generalized Born Surface Area (MM-GBSA) method (Kollman et al. 2000), which can be conceptually summarized as the following equations:

$$
\Delta \mathrm{G}_{\text {bind }}=\Delta \mathrm{G}_{\text {complex }}-\Delta \mathrm{G}_{\text {protein }}-\Delta \mathrm{G}_{\text {ligand }}
$$

and

$$
\Delta \mathrm{G}_{\text {binding }}=\Delta \mathrm{G}_{\mathrm{MM}}+\Delta \mathrm{G}_{\mathrm{SOL}}-\mathrm{T} \Delta \mathrm{S}
$$

where $\Delta \mathrm{G}_{\text {complex }}, \Delta \mathrm{G}_{\text {protein }}$, and $\Delta \mathrm{G}_{\text {ligand }}$ are the free energies of the complex, the protein, and the ligand, respectively. Each of them is calculated by summing the total molecular mechanical energy $\left(\Delta \mathrm{G}_{\mathrm{MM}}\right)$, the solvation free energy $\left(\Delta \mathrm{G}_{\mathrm{SOL}}\right)$, and the entropy $(\mathrm{T} \Delta \mathrm{S})$. The molecular mechanical energy has three energetic terms: an electrostatic term, a van der Waals term, and an internal energy term. The solvation free energy is divided into two contributions: the polar $\left(\Delta \mathrm{G}_{\mathrm{PB} / \mathrm{GB}}\right)$ and nonpolar $\left(\Delta \mathrm{G}_{\mathrm{NP}}\right)$ components.

The main goal of this section prefers to identify residues that play a dominant role in binding of the pre-miRNA to the complex Exp5-GTP rather than acquire a quantitative account of the binding thermodynamics; thus, we neglect conformational change upon complexation. In other words, we neglect the internal energy terms and conformational entropy contributions to the thermodynamics of binding. Notably, although it is possible to analyze individual contributions to conformational entropies (Fischer et al. 2001), the simplification saves the significant and unnecessary computational cost. Moreover, it has been applied successfully in the context of identifying interaction energy "hot spots" in antigen-antibody complexes (Lafont et al. 2007). Therefore, in this section, we calculated only a subset of terms entering the MM-GBSA, i.e., the electrostatic, the van der Waals, and the hydrophobic contributions. Then the calculation of binding of free energy is according to the following equation:

$$
\Delta \mathrm{G}=\Delta \mathrm{E}^{\text {elec }}+\Delta \mathrm{E}^{\mathrm{vdw}}+\Delta \mathrm{G}_{\text {solv }}^{\text {ele }}+\Delta \mathrm{G}_{\text {solv }}^{\text {np }}
$$

where $\Delta \mathrm{E}^{\text {elec }}$ and $\Delta \mathrm{E}^{\mathrm{vdw}}$ are the electrostatic and van der Waals contributions to the energy of the complex formation, respectively; and $\Delta \mathrm{G}_{\text {solv }}^{\text {elec }}$ and $\Delta \mathrm{G}_{\text {solv }}^{\text {np }}$ are the electrostatic and nonpolar contributions related to solvation, respectively.

The binding free energy decomposition of the Exp5-GTP-RNA complex was analyzed using the AMBER 10 package (Pearlman 
et al. 1995) to evaluate the contribution of each residue to the total binding free energy, as well as the contributions of its side chain and backbone. Firstly, the polar component of the solvation free energy was computed with the GB approximation model (Kollman et al. 2000). The contribution of atom $i$ to the total electrostatic interaction energy is represented by:

$$
\mathrm{G}_{\text {elec }}^{\mathrm{i}}=\frac{1}{2} \sum_{\mathrm{j} \neq \mathrm{i}} \frac{\mathrm{q}_{\mathrm{i}} \mathrm{q}_{\mathrm{j}}}{\mathrm{r}_{\mathrm{ij}}}-\frac{1}{2} \sum_{\mathrm{j}}\left(1-\frac{\mathrm{e}^{-\lambda \mathrm{f}_{\mathrm{GB}}}}{\varepsilon_{\omega}}\right)
$$

and

$$
f_{G B}=\sqrt{r_{i j}^{2}+\alpha_{i} \alpha_{j} \exp \left(\frac{-r_{i j}^{2}}{4 \alpha_{i} \alpha_{j}}\right)}
$$

where $\mathrm{q}_{\mathrm{i}}$ and $\mathrm{q}_{\mathrm{j}}$ are atomic partial charges, $\varepsilon_{\omega}$ the solvent dielectric constant, $\lambda$ the Debye-Huckel screening parameter, and the double sum runs over all pairs of atoms. $r_{i j}$ is the distance between atom $i$ and atom $j . \alpha_{i}$ and $\alpha_{j}$ are the effective Born radius of atoms $i$ and $j$, respectively. Secondly, one-half of the pairwise van der Waals interaction energy is added to $G^{i}$ to which the atom belongs that is part of the interaction pair (Gohlke et al. 2003). Thirdly, the nonpolar solvation part was obtained by fitting the solvent-accessible surface area (SASA) (Weiser et al. 1999). Finally, to obtain the contribution of each residue, as well as the contributions of its side chain and backbone, we summed the contribution of relevant atoms. A total of 50 snapshots were extracted evenly from the last $0.5 \mathrm{nsec}$ on the MD trajectory with an interval of $10 \mathrm{psec}$ to average the energy term. For the sake of convenient comparison, we analyzed the contribution of the premiRNA nucleotides to the binding by determining their individual relative contribution ratio:

$$
\mathrm{R}=\frac{\Delta \mathrm{G}_{\mathrm{N}}}{\Delta \mathrm{G}_{\mathrm{RNA}}} \times 100 \%
$$

where $\Delta \mathrm{G}_{\mathrm{N}}$ indicates the total energetic contribution of individual nucleotides to the binding free energy, and $\Delta G_{R N A}$ represents the total energetic contribution of pre-miRNA to binding free energy.

\section{Water density analysis}

The approach to obtain the density map from the MD trajectory has been described in greater detail elsewhere (Higo et al. 2000). According to this, the computed high-density sites of the solvent around the protein are well correlated with the experimentally determined crystal-water sites. Here, we describe the approach briefly: Firstly, we calculated the average apo-Exp5 conformation over the trajectory by superimposing the protein conformations in the last $5 \mathrm{nsec}$ of the trajectory on the initial structure. The reference conformation was defined as the structure with the smallest RMSD from the average conformation. Secondly, the atom coordinates were transformed by superimposing the protein conformations in the last 5-nsec trajectory onto a reference model. The solvent molecules were translated and rotated together with the protein conformations. Thirdly, the solvent density map of apoExp5 from the MD trajectory was estimated from the water oxygen atom positions as described by Lounnas and Pettitt (1994a,b). Based on these, the water distribution map was obtained.

\section{SMD simulations}

SMD is an extended MD simulation method mimicking the basic idea of atomic force microscopy (AFM). SMD simulations, furthermore, have been applied successfully to explore the binding and unbinding properties of a variety of biomolecules as well as their responses to external mechanical manipulations at the atomic level (Jarzynski 1997; Liphardt et al. 2002). It is particularly suitable for the investigation of the dissociation of a ligand from its binding protein. In the present SMD simulations, only Exp5-GTP-RNA and Exp5-RNA were simulated to uncover the mechanism of how pre-miRNA is released and whether the binding of RanGTP can affect the dissociation process. Before executing the actual SMD simulations, several directions for the pulling force were identified based on the relative orientation of target RNA and its binding site. Then four independent SMD simulations for each system were performed, in which the direction of the pulling force was chosen in the range of possible directions just identified. The complexes were incubated in boxes of TIP3P-minimized water with dimensions $18.632 \times 16.632 \times$ $14.632 \mathrm{~nm}^{3}$, which is 1.5 times larger than the original water box to ensure that the SMD simulation was more feasible. Each system was applied with four different forces independently; all these forces were acting along the RNA axis direction. The H12A, $\mathrm{H} 13 \mathrm{~A}$, and H14A, which are located in the convex and bottom of Exp5, were fixed in space to hold the complex in a proper position during the SMD simulations. Harmonic constant forces of 1000 , $2000,10,000$, and $11,000 \mathrm{~kJ} / \mathrm{mol}$ per $\AA^{2}$ were applied to each system, resulting in a total of 36-nsec simulations. The stretch distance between pre-miRNA and Exp5 was defined as the change of the distance between the center of mass (COM) of base pair G1/C61 and the COM of H13B (residues 641-657) (Fig. 1A).

\section{SUPPLEMENTAL MATERIAL}

Supplemental material is available for this article. Additional color figures can be found at: http://goo.gl/thigg.

\section{ACKNOWLEDGMENTS}

We are grateful to Professor Ling Yang for access to the Sybyl software. And we are also grateful to Professor Monika Walter for an excellent and professional revision of the English of our manuscript. This work is supported by the high-performance computing platform of Northwest A\&F University, and is financially supported by the National Natural Science Foundation of China (Grant No. 10801025) and also the Fund of Northwest A\&F University.

Received March 17, 2011; accepted May 19, 2011.

\section{REFERENCES}

Amadei A, Linnsen AB, Berendsen HJ. 1993. Essential dynamics of proteins. Proteins 17: 412-425.

Bartel DP. 2004. MicroRNAs: Genomics, biogenesis, mechanism, and function. Cell 116: 281-297.

Berendsen HJC, Postma JPM, van Gunsteren WF, Di Nola A, Haak JR. 1984. Molecular dynamics with coupling to an external bath. J Chem Phys 81: 3684-3690. 
Bernstein E, Caudy AA, Hammond SM, Hannon GJ. 2001. Role for a bidentate ribonuclease in the initiation step of RNA interference. Nature 409: 363-366.

Bischoff FR, Görlich D. 1997. RanBP1 is crucial for the release of RanGTP from importin-related nuclear transport factors. FEBS Lett 419: 249-254.

Bohnsack MT, Czaplinski K, Gorlich D. 2004. Exportin 5 is a RanGTP-dependent dsRNA-binding protein that mediates nuclear export of pre-miRNAs. RNA 10: 185-191.

Brownawell AM, Macara IG. 2002. Exportin-5, a novel karyopherin, mediates nuclear export of double-stranded RNA binding proteins. J Cell Biol 156: 53-64.

Bullock SL, Ringel I, Ish-Horowicz D, Lukavsky PJ. 2010. A'-form RNA helices are required for cytoplasmic mRNA transport in Drosophila. Nat Struct Mol Biol 17: 703-710.

Caves LS, Evanseck JD, Karplus M. 1998. Locally accessible conformations of proteins: Multiple molecular dynamics simulations of crambin. Protein Sci 7: 649-666.

Conti E, Müller CW, Stewart M. 2006. Karyopherin flexibility in nucleocytoplasmic transport. Curr Opin Struct Biol 16: 237-244.

Cook A, Fernandez E, Lindner D, Ebert J, Schlenstedt G, Conti E. 2005. The structure of the nuclear export receptor Csel in its cytosolic state reveals a closed conformation incompatible with cargo binding. Mol Cell 18: 355-367.

Cook A, Fukuhara N, Jinek M, Conti E. 2009. Structures of the tRNA export factor in the nuclear and cytosolic states. Nature 461: 60-65.

Darden T, York D, Pedersen L. 1993. Particle mesh Ewald: An $N \cdot \log (N)$ method for Ewald sums in large systems. J Chem Phys 98: 10089-10092.

Duan J, Nilsson L. 2002. The role of residue 50 and hydration water molecules in homeodomain DNA recognition. Eur Biophys J 31: 306-316.

Duan Y, Wu C, Chowdhury S, Lee MC, Xiong G, Zhang W, Yang R, Cieplak P, Luo R, Lee T, et al. 2003. A point-charge force field for molecular mechanics simulations of proteins based on condensedphase quantum mechanical calculations. J Comput Chem 24: 1999-2012.

Fischer S, Smith JC, Verma CS. 2001. Dissecting the vibrational entropy change on protein/ligand binding: Burial of a water molecule in BPTI. J Phys Chem B 105: 8050-8055.

Fried H, Kutay U. 2003. Nucleocytoplasmic transport: taking an inventory. Cell Mol Life Sci 60: 1659-1688.

Gohlke H, Kiel C, Case DA. 2003. Insights into protein-protein binding by binding free energy calculation and free energy decomposition for the Ras-Raf and Ras-RalGDS complexes. J Mol Biol 330: 891-913.

Hayward S, Berendsen HJ. 1998. Systematic analysis of domain motions in proteins from conformational change: New results on citrate synthase and T4 lysozyme. Proteins 30: 144-154.

Hess B, Bekker H, Berendsen HJC, Fraaije JGE. 1997. LINCS: A linear constraint solver for molecular simulations. J Comput Chem 18: 1463-1472.

Hess B, Kutzner C, Van Der Spoel D, Lindahl E. 2008. GROMACS 4: Algorithms for highly efficient, load-balanced, and scalable molecular simulation. J Chem Theory Comput 4: 435-447.

Higo J, Kono H, Nakamura H, Sarai A. 2000. Solvent density and long-range dipole field around a DNA-binding protein studied by molecular dynamics. Proteins 40: 193-206.

Ivetac A, McCammon JA. 2009. Elucidating the inhibition mechanism of HIV-1 non-nucleoside reverse transcriptase inhibitors through multicopy molecular dynamics simulations. J Mol Biol 388: 644658.

Jarzynski C. 1997. Nonequilibrium equality for free energy differences. Phys Rev Lett 78: 2690-2693.

Jorgensen WL, Chandrasekhar J, Madura JD, Klein ML. 1983. Comparison of simple potential functions for simulating liquid water. J Chem Phys 79: 926-935.

Kehlenbach RH, Dickmanns A, Kehlenbach A, Guan T, Gerace L. 1999. A role for RanBP1 in the release of CRM1 from the nuclear pore complex in a terminal step of nuclear export. J Cell Biol 145: 645-657.

Kollman PA, Massova I, Reyes C, Kuhn B, Huo S, Lee M, Lee T, Duan Y, Wang W, Donini O, et al. 2000. Calculating structures and free energies of complex molecules: Combining molecular mechanics and continuum models. Acc Chem Res 33: 889-897.

Lafont V, Schaefer M, Stote RH, Altschuh D, Dejaegere A. 2007. Protein-protein recognition and interaction hot spots in an antigen-antibody complex: Free energy decomposition identifies "efficient amino acids." Proteins 67: 418-434.

Lee Y, Jeon K, Lee JT, Kim SY, Kim VN. 2002. MicroRNA maturation: stepwise processing and subcellular localization. EMBO $J$ 21: 4663-4670.

Lee Y, Ahn C, Han J, Choi H, Kim J, Yim J, Lee J, Provost P, Radmark O, Kim S, et al. 2003. The nuclear RNase III Drosha initiates microRNA processing. Nature 425: 415-419.

Leijon M, Graslund A. 1992. Effects of sequence and length on imino proton-exchange and basepair opening kinetics in DNA oligonucleotide duplexes. Nucleic Acids Res 20: 5339-5343.

Liphardt J, Dumont S, Smith SB, Tinoco I, Bustamante C. 2002. Equilibrium information from nonequilibrium measurements in an experimental test of Jarzynski's equality. Science 296: 18321835.

Lounnas V, Pettitt BM. 1994a. A connected-cluster of hydration around myoglobin: Correlation between molecular dynamics simulations and experiment. Proteins 18: 133-147.

Lounnas V, Pettitt BM. 1994b. Distribution function implied dynamics versus residence times and correlations: Solvation shells of myoglobin. Proteins 18: 148-160.

Lu XJ, Olson WK. 2003. 3DNA: a software package for the analysis, rebuilding and visualization of three-dimensional nucleic acid structures. Nucleic Acids Res 31: 5108-5121.

Lund E, Guttinger S, Calado A, Dahlberg JE, Kutay U. 2004. Nuclear export of microRNA precursors. Science 303: 95-98.

Lynch TW, Kosztin D, McLean MA, Schulten K, Sligar SG. 2002. Dissecting the molecular origins of specific protein-nucleic acid recognition: Hydrostatic pressure and molecular dynamics. Biophys J 82: 93-98.

MacDonald D, Demarre G, Bouvier M, Mazel D, Gopaul DN. 2006. Structural basis for broad DNA-specificity in integron recombination. Nature 440: 1157-1162.

MacKerell AD Jr, Nilsson L. 2008. Molecular dynamics simulations of nucleic acid-protein complexes. Curr Opin Struct Biol 18: 194-199.

Meagher KL, Redman LT, Carlson HA. 2003. Development of polyphosphate parameters for use with the AMBER force field. J Comput Chem 24: 1016-1025.

Nandi N, Bhattacharyya K, Bagchi B. 2000. Dielectric relaxation and solvation dynamics of water in complex chemical and biological systems. Chem Rev 100: 2013-2045.

Okada C, Yamashita E, Lee SJ, Shibata S, Katahira J, Nakagawa A, Yoneda Y, Tsukihara T. 2009. A high-resolution structure of the pre-microRNA nuclear export machinery. Science 326: 1275-1279.

Ossareh-Nazari B, Bachelerie F, Dargemont C. 1997. Evidence for a role of CRM1 in signal-mediated nuclear protein export. Science 278: 141-144.

Pace CN, Shirley BA, McNutt M, Gajiwala K. 1996. Forces contributing to the conformational stability of proteins. FASEB J 10: 75-83.

Pearlman DA, Case DA, Caldwell JW, Ross WS, Cheatham TE III, DeBolt S, Ferguson D, Seibel GL, Kollman PA. 1995. AMBER, a package of computer programs for applying molecular mechanics, normal mode analysis, molecular dynamics and free energy calculations to simulate the structural and energetic properties of molecules. Comput Phys Commun 91: 1-41.

Phillips JC. 2008. Scaling and self-organized criticality in proteins II. Proc Natl Acad Sci 106: 3113-3118.

Phillips GN Jr, Pettitt BM. 1995. Structure and dynamics of the water around myoglobin. Protein Sci 4: 149-158.

Reddy CK, Das A, Jayaram B. 2001. Do water molecules mediate protein-DNA recognition? J Mol Biol 314: 619-632. 
Wang et al.

Schreiber G, Fersht AR. 1995. Energetics of protein-protein interactions: Analysis of the Barnase-Barstar interface by single mutations and double mutant cycles. J Mol Biol 248: 478-486.

Schwabe JW. 1997. The role of water in protein-DNA interactions. Curr Opin Struct Biol 7: 126-134.

Schwarz DS, Hutvagner G, Du T, Xu Z, Aronin N, Zamore PD. 2003. Asymmetry in the assembly of the RNAi enzyme complex. Cell 115: 199-208.

Snoussi K, Leroy JL. 2001. Imino proton exchange and base-pair kinetics in RNA duplexes. Biochemistry 40: 8898-8904.

Stade K, Ford CS, Guthrie C, Weis K. 1997. Exportin 1 (Crmlp) is an essential nuclear export factor. Cell 90: 1041-1050.

Teeter MM, Yamano A, Stec B, Mohanty U. 2001. On the nature of a glassy state of matter in a hydrated protein: Relation to protein function. Proc Natl Acad Sci 98: 11242-11247.

Vallone B, Miele AE, Vecchini P, Chiancone E, Brunori M. 1998. Free energy of burying hydrophobic residues in the interface between protein subunits. Proc Natl Acad Sci 95: 6103-6107.

van Aalten DM, Amadei A, Linssen AB, Eijsink VG, Vriend G, Berendsen HJ. 1995a. The essential dynamics of thermolysin: Confirmation of the hinge-bending motion and comparison of simulations in vacuum and water. Proteins 22: 45-54.

van Aalten DM, Findlay JB, Amadei A, Berendsen HJ. 1995b. Essential dynamics of the cellular retinol-binding protein-evidence for ligand-induced conformational changes. Protein Eng 8: 11291135.
Vriend G. 1990. WHAT IF: A molecular modeling and drug design program. J Mol Graph 8: 52-56.

Wang Y, Li Y, Ma Z, Yang W, Ai C. 2010a. Mechanism of microRNAtarget interaction: Molecular dynamics simulations and thermodynamics analysis. PloS Comput Biol 6: e1000866. doi: 10.1371/ journal.pcbi.1000866.

Wang X, Li Y, Xu X, Wang Y. 2010b. Toward a system-level understanding of microRNA pathway via mathematical modeling. Biosystems 100: 31-38.

Weis K. 2003. Regulating access to the genome: Nucleocytoplasmic transport throughout the cell cycle. Cell 112: 441-451.

Weiser J, Shenkin PS, Still WC. 1999. Approximate atomic surfaces from linear combinations of pairwise overlaps (LCPO). J Comput Chem 20: 217-230.

Yi R, Qin Y, Macara IG, Cullen BR. 2003. Exportin-5 mediates the nuclear export of pre-microRNAs and short hairpin RNAs. Genes Dev 17: 3011-3016.

Zachariae U, Grubmüller H. 2006. A highly strained nuclear conformation of the exportin Cselp revealed by molecular dynamics simulations. Structure 14: 1469-1478.

Zeng Y, Cullen BR. 2004. Structural requirements for pre-microRNA binding and nuclear export by Exportin 5. Nucleic Acids Res 32: 4776-4785.

Zeng Y, Wagner EJ, Cullen BR. 2002. Both natural and designed micro RNAs can inhibit the expression of cognate mRNAs when expressed in human cells. Mol Cell 9: 1327-1333. 

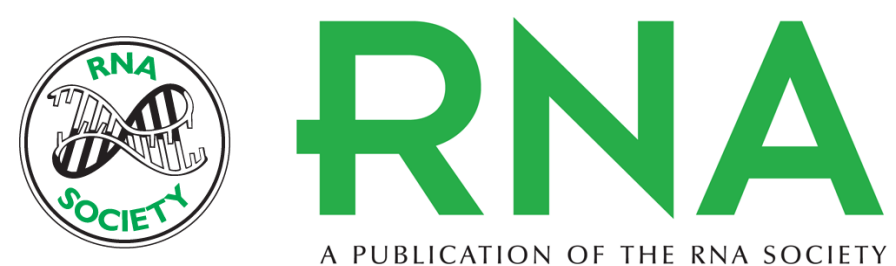

A PUBLICATION OF THE RNA SOCIETY

\section{Dynamic mechanisms for pre-miRNA binding and export by Exportin-5}

Xia Wang, Xue Xu, Zhi Ma, et al.

RNA 2011 17: 1511-1528 originally published online June 28, 2011

Access the most recent version at doi:10.1261/rna.2732611

Supplemental

Material

References

\section{License}

Email Alerting Service
http://rnajournal.cshlp.org/content/suppl/2011/06/06/rna.2732611.DC1

This article cites 66 articles, 11 of which can be accessed free at: http://rnajournal.cshlp.org/content/17/8/1511.full.html\#ref-list-1

\section{top right corner of the article or click here.}

Receive free email alerts when new articles cite this article - sign up in the box at the

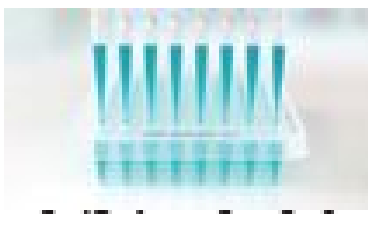

